\title{
The Simons Observatory Large Aperture Telescope Receiver
}

\author{
Ningfeng Zhu ${ }^{1}(\mathbb{1})$, Tanay Bhandarkar ${ }^{1}$ (1), Gabriele Coppi ${ }^{1,2}$, Anna M. Kofman ${ }^{1}$, John L. Orlowski-Scherer ${ }^{1}$ (1), Zhilei Xu ${ }^{1,3}$ (1),
} Shunsuke Adachi ${ }^{4}$, Peter Ade ${ }^{5}$ (1), Simone Aiola ${ }^{6}$ (1) Jason Austermann $^{7}$, Andrew O. Bazarko ${ }^{8}$, James A. Beall ${ }^{7}$, Sanah Bhimani ${ }^{9}$, J. Richard Bond ${ }^{10}$, Grace E. Chesmore ${ }^{11}$, Steve K. Choi $^{12,13}$ (D), Jake Connors 7 (1), Nicholas F. Cothard ${ }^{14}$ (1) , Mark Devlin $^{1}$ (1) Simon Dicker ${ }^{1}(\mathbb{1})$, Bradley Dober ${ }^{15}$ (1) , Cody J. Duell $^{12}$, Shannon M. Duff ${ }^{7}$ (i), Rolando Dünner ${ }^{16}$ (1), Giulio Fabbian ${ }^{5}$ (1)

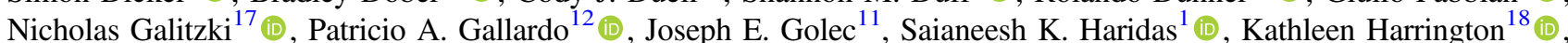
Erin Healy ${ }^{8}$, Shuay-Pwu Patty Ho ${ }^{19}$, Zachary B. Huber ${ }^{12}$ (i), Johannes Hubmayr ${ }^{7}$, Jeffrey Iuliano ${ }^{1}$ (i), Bradley R. Johnson ${ }^{20}$ (i), Brian Keating ${ }^{17}$, Kenji Kiuchi ${ }^{21}$ (1), Brian J. Koopman ${ }^{9}$ (i), Jack Lashner ${ }^{22}$, Adrian T. Lee ${ }^{23}$, Yaqiong Li ${ }^{12,24}$, Michele Limon ${ }^{1}$ (1) , Michael Link ${ }^{7}$, Tammy J Lucas ${ }^{7}$ (1) , Heather McCarrick ${ }^{8}$, Jenna Moore ${ }^{25}$ (10), Federico Nati ${ }^{2}$ (1), Laura B. Newburgh ${ }^{26}$, Michael D. Niemack ${ }^{12,13,24}$ (1) , Elena Pierpaoli ${ }^{22}$, Michael J. Randall ${ }^{17}$, Karen Perez Sarmiento ${ }^{1}$, Lauren J. Saunders ${ }^{9}$, Joseph Seibert $^{17}$, Carlos Sierra ${ }^{11}$, Rita Sonka ${ }^{8}$, Jacob Spisak ${ }^{17}$ (1), Shreya Sutariya ${ }^{11}$, Osamu Tajima ${ }^{4}$, Grant P. Teply ${ }^{17}$, Robert J. Thornton ${ }^{1,27}$, Tran $\operatorname{Tsan}^{17}$ (D), Carole Tucker $^{5}$ (D), Joel Ullom ${ }^{7}$, Eve M. Vavagiakis ${ }^{12}$, Michael R. Vissers ${ }^{7}$ (1),

Samantha Walker ${ }^{7,28}$ (i), Benjamin Westbrook ${ }^{23}$, Edward J. Wollack ${ }^{29}$ (i), and Mario Zannoni ${ }^{2}$ (i)

${ }^{1}$ Department of Physics and Astronomy, University of Pennsylvania, 209 South 33rd Street, Philadelphia, PA 19104, USA; nin@ sas.upenn.edu

${ }^{2}$ Department of Physics, University of Milano-Bicocca, Milano (MI), Italy

${ }^{3}$ MIT Kavli Institute, Massachusetts Institute of Technology, 77 Massachusetts Avenue, Cambridge, MA 02139, USA

${ }^{4}$ Department of Physics, Kyoto University, Kitashirakawa Oiwake-cho, Sakyo-ku, Kyoto 606-8502, Japan

${ }^{5}$ School of Physics and Astronomy, Cardiff University, The Parade, Cardiff, CF24 3AA, UK

${ }^{6}$ Center for Computational Astrophysics, Flatiron Institute, 162 5th Avenue, New York, NY 10010, USA

${ }^{7}$ NIST Quantum Sensors Group, 325 Broadway Ave, Boulder, CO 80305, USA

${ }^{8}$ Department of Physics, Princeton University, Princeton, NJ 08540, USA

${ }^{9}$ Department of Physics, Yale University, New Haven, CT 06520, USA

${ }^{10}$ CITA, University of Toronto, Toronto, ON M5S 3H8, Canada

${ }^{11}$ Department of Physics, University of Chicago, Chicago, IL 60637, USA

12 Department of Physics, Cornell University, Ithaca, NY 14853, USA

${ }^{13}$ Department of Astronomy, Cornell University, Ithaca, NY 14853, USA

${ }^{14}$ Department of Applied and Engineering Physics, Cornell University, Ithaca, NY 14853, USA
${ }^{15}$ Department of Physics, University of Colorado Boulder, Department of Physics 390, Boulder, CO 80309, USA

${ }^{16}$ Instituto de Astrofísica and Centro de Astro-Ingeniería, Facultad de Física, Pontificia Universidad Católica de Chile, Av. Vicuña Mackenna 4860, 7820436, Macul, Santiago, Chile

${ }^{17}$ Department of Physics, University of California San Diego, La Jolla, CA 92093, USA

${ }^{18}$ Department of Astronomy and Astrophysics, University of Chicago, Chicago, IL 60637, USA

${ }^{19}$ Department of Physics, Stanford University, Stanford, CA 94305, USA

${ }^{20}$ Department of Astronomy, University of Virginia, Charlottesville, VA 22904, USA

${ }^{21}$ Department of Physics, The University of Tokyo, 7-3-1 Hongo, Bunkyo, Tokyo 113-0033, Japan

${ }^{22}$ Department of Physics and Astronomy, University of Southern California, Los Angeles, CA 90089, USA

${ }^{23}$ Physics Department, University of California, Berkeley, CA 94720, USA

${ }^{24}$ Kavli Institute at Cornell for Nanoscale Science, Cornell University, Ithaca, NY 14853, USA

${ }^{25}$ School of Earth and Space Exploration, Arizona State University, Tempe, AZ 85287, USA

${ }^{27}$ Department of Physics and Engineering, West Chester University of Pennsylvania, West Chester, PA 19383, USA

${ }^{28}$ Department of Astrophysical and Planetary Sciences, University of Colorado Boulder, Boulder, CO 80309, USA

${ }^{29}$ NASA Goddard Space Flight Center, Greenbelt, MD 20771, USA

Received 2021 March 4; revised 2021 May 19; accepted 2021 June 21; published 2021 September 16

\begin{abstract}
The Simons Observatory is a ground-based cosmic microwave background experiment that consists of three $0.4 \mathrm{~m}$ small-aperture telescopes and one $6 \mathrm{~m}$ Large Aperture Telescope, located at an elevation of $5300 \mathrm{~m}$ on Cerro Toco in Chile. The Simons Observatory Large Aperture Telescope Receiver (LATR) is the cryogenic camera that will be coupled to the Large Aperture Telescope. The resulting instrument will produce arcminute-resolution millimeterwave maps of half the sky with unprecedented precision. The LATR is the largest cryogenic millimeter-wave camera built to date, with a diameter of $2.4 \mathrm{~m}$ and a length of $2.6 \mathrm{~m}$. The coldest stage of the camera is cooled to $100 \mathrm{mK}$, the operating temperature of the bolometric detectors with bands centered around 27, 39, 93, 145, 225, and $280 \mathrm{GHz}$. Ultimately, the LATR will accommodate $1340 \mathrm{~cm}$ diameter optics tubes, each with three detector wafers and a total of 62,000 detectors. The LATR design must simultaneously maintain the optical alignment of the system, control stray light, provide cryogenic isolation, limit thermal gradients, and minimize the time to cool the system from room temperature to $100 \mathrm{mK}$. The interplay between these competing factors poses unique challenges. We discuss the trade studies involved with the design, the final optimization, the construction, and ultimate performance of the system.
\end{abstract}

Unified Astronomy Thesaurus concepts: CMBR detectors (259); Cosmic microwave background radiation (322); Observational cosmology (1146); Ground-based astronomy (686); Ground telescopes (687); Observatories (1147); Astronomical instrumentation (799) 


\section{Introduction}

Observations of the cosmic microwave background (CMB) are crucial tools in developing our understanding of the physics of the early universe and testing the standard model of cosmology, $\Lambda$ CDM (Condon \& Matthews 2018). While satellite missions such as the Cosmic Background Explorer (COBE; Smoot 1999), the Wilkinson Microwave Anisotropy Probe (WMAP; Bennett et al. 2013; Hinshaw et al. 2013), and the Planck Collaboration (Planck Collaboration et al. 2020) have produced full-sky microwave maps, ground-based experiments have extended the satellite measurements toward smaller angular scales and lower noise levels over $40 \%$ the sky. High-resolution $\left(\sim 1^{\prime}\right)$ experiments, such as the Atacama Cosmology Telescope (ACT; Thornton et al. 2016) and the South Pole Telescope (SPT; Carlstrom et al. 2011; Sayre et al. 2020) have made measurements of both the primordial temperature power spectrum (Wu et al. 2019) as well as secondary anisotropies such as the thermal and kinematic Sunyaev-Zel'dovich (SZ) effects (Sunyaev \& Zeldovich 1970, 1972; Hilton et al. 2018) and gravitational lensing effects (Story et al. 2015). Lowresolution experiments $\left(\sim 0^{\circ} .5\right)$, such as the BICEP and Keck Arrays (Ade et al. 2014a; Keck Array and BICEP2 Collaborations et al. 2018), the SPIDER (Gualtieri et al. 2018), the Atacama B-mode Survey (ABS; Kusaka et al. 2018), the POLARBEAR (Ade et al. 2014b; Polarbear Collaboration et al. 2020), and the Cosmology Large Angular Scale Surveyor (CLASS; Harrington et al. 2016; Xu et al. 2020) aim to improve measurements on B-mode polarization, one of the two components in CMB polarization (Hu \& White 1997), at larger angular scales $(\ell \lesssim 200)$.

The Simons Observatory (SO; Galitzki et al. 2018; The Simons Observatory Collaboration 2019) is a next-generation CMB experiment consisting of three $0.42 \mathrm{~m}$ small-aperture telescopes (SATs; Ali et al. 2020) and one $6 \mathrm{~m}$ Large Aperture Telescope (LAT; Parshley et al. 2018) in the Atacama Desert of Chile. The LAT utilizes a crossed-Dragone optical design with a pair of $6 \mathrm{~m}$ mirrors (Gallardo et al. 2018; Gudmundsson et al. 2021). The Large Aperture Telescope Receiver (LATR; Orlowski-Scherer et al. 2018; Zhu et al. 2018; Xu et al. 2020) can contain up to 13 optics tubes (OTs) and will cover six frequency bands centered around 27, 39, 93, 145, 225, and $280 \mathrm{GHz}$ with polarization-sensitive dichroic pixels. The OT is a self-contained unit including components at $4 \mathrm{~K}, 1 \mathrm{~K}$, and $100 \mathrm{mK}$. Its detailed design is discussed in Section 3.3. The initial survey will use seven OTs in the nominal configuration, with the remaining space allowing for future upgrades. Each OT contains $\sim 5000$ (depending on the frequency band) polarizationsensitive transition edge sensor (TES) detectors (Irwin \& Hilton 2005) operating below $100 \mathrm{mK}$. The LATR is $2.6 \mathrm{~m}$ in length, $2.4 \mathrm{~m}$ in diameter, and $11 \mathrm{~m}^{3}$ in volume. It is installed on a bore-sight rotation mount in the LATR cabin, where it uses a 6.7 field of view out of the 7.8 available.

A detailed design study was performed to optimize the overall sensitivity of the instrument while considering the technical challenges and timeline of the program. For example, the size of the focal plane on the LAT could accommodate a 19 tube LATR, but this was deemed too technically risky and a 13 OT LATR was chosen as the best balance between meeting the near-term goals of SO while allowing for significant future expansion. Likewise, a $100 \mathrm{mK}$ dilution refrigerator provides continuous operation and increased sensitivity over a $300 \mathrm{mK} \mathrm{He}-3$ adsorption-cooled system (Thornton et al. 2016; Abazajian et al. 2019). Such a system has been successfully operating on ACT since 2011 .

Over a five-year observing period, we plan to use the LAT to survey $\sim 40 \%$ of the sky with arcminute resolution and a map noise level of $\sim 6 \mu \mathrm{K}$-arcmin in the 93 and $145 \mathrm{GHz}$ bands (see Table 1). Overlapping with existing and upcoming optical surveys, the $\mathrm{SO}$ will be able to measure cross-correlations of the SO-reconstructed lensing potential with the Vera C. Rubin Observatory (Ivezić et al. 2019) identified galaxies, and provide tighter constraints on the neutrino mass by combining SO data with Dark Energy Spectroscopic Instrument (DESI; DESI Collaboration et al. 2016) baryon acoustic oscillation (BAO) information. The LAT will be able to make precise measurements of the small-scale temperature and polarization power spectra, as well as the CMB lensing spectrum (The Simons Observatory Collaboration 2019). Additionally, the LAT will detect tens of thousands of clusters using the thermal SZ effect, as well as numerous extragalactic sources and transient microwave objects (Naess et al. 2021; Guns et al. 2021). There are even prospects for the LAT to detect Oort clouds around nearby stars (Blake et al. 2019; Orlowski-Scherer et al. 2019) or additional planets in our solar system (Baxter et al. 2018). Finally, observations made with the LAT can be used to delens largescale B-mode signals (Ade et al. 2021) measured with the SATs. A complete discussion of the LAT science can be found in the SO forecast paper (The Simons Observatory Collaboration 2019).

In this paper, we start with a design overview for the LATR in Section 2. Then, the detailed design of the LATR is discussed in Section 3. The LATR testing and validation results are presented in Section 4. Finally, we summarize the lessons for future development in Section 5, and present our conclusions in Section 6.

\section{Design Overview}

The LAT utilizes a cross-Dragone design with a pair of $6 \mathrm{~m}$ aperture mirrors. The $3 \mathrm{~mm}$ wavelength diffraction-limited focal plane is $1.9 \mathrm{~m}$ in diameter with a 7.8 field of view (Thornton et al. 2016; Parshley et al. 2018). While the LATR could have been designed to fill the entire LAT focal plane, we choose to limit the used part of the focal plane to $1.7 \mathrm{~m}$ diameter and a 6.7 field of view out of the $7^{\circ} .8$ available. The LATR is shown installed in the LAT in Figure 1. A photo of the LATR being tested at the University of Pennsylvania is shown in Figure 2.

Inside the cryostat, cryogenic optics within the OTs reimage the telescope focal plane onto the detector arrays. The detectors are packaged into three universal focal plane modules (UFM), which are cooled to below $100 \mathrm{mK}$ to improve the detector sensitivity. Metal mesh filters and alumina filters modulate the thermal loading. An advantage of the modularized OT design is that each OT can be optimized for a specific frequency range, as shown in Table 1.

A trade-off study was performed to select the number of OTs for the LATR, with the aim of optimizing sensitivity while accounting for mechanical, optical, and cryogenic constraints (Hill et al. 2018). Smaller OTs allow for smaller optical elements, which are easier to fabricate. However, one then needs more OTs to fill the same focal plane area, which adds mass and reduces the filling factor due to required spacing for the magnetic shielding, mechanical structures, and cryogenic connections. The additional mass also requires a thicker front plate, which in turn increases the OT separation to avoid 


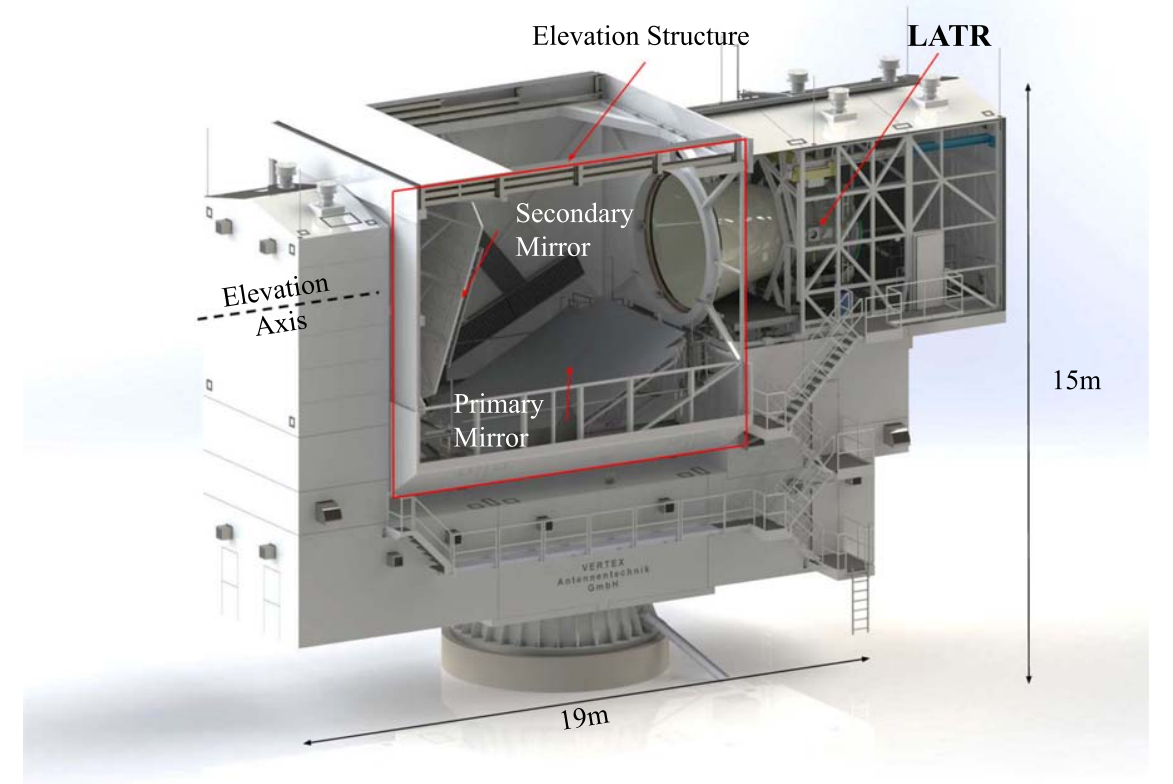

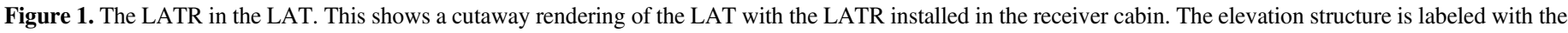

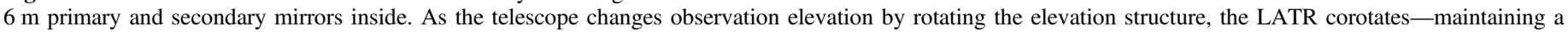
consistent orientation between the LATR and the primary and secondary mirrors.

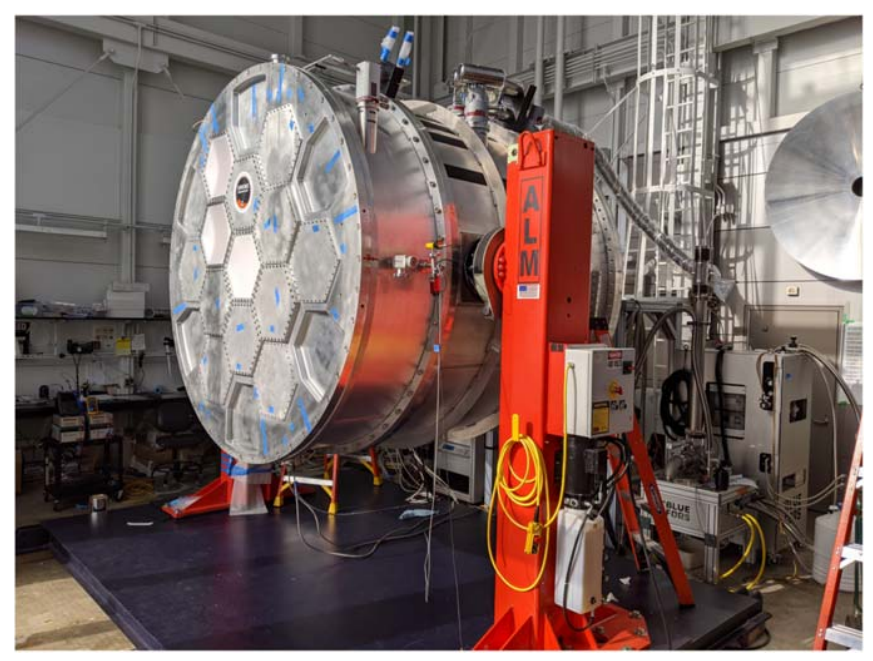

Figure 2. The LATR in the highbay at the University of Pennsylvania. The photo shows the LATR during testing. The $2.4 \mathrm{~m}$ diameter front plate with 13 hexagonal openings for optics tubes can be seen in the picture. The LATR is held by two arms (red) on a platform (dark blue). The arms are also capable of rotating the LATR with an installed motor.

clipping the beams exiting the LATR. On the other hand, if OTs are too large in diameter, the filters will be much harder to manufacture. The research and development time needed for large filter fabrication could potentially delay the project schedule. Balancing the required size of the optical elements with the overhead of additional tubes leads us to an optimum of 13 OTs.

At 5 metric tons with a $2.4 \mathrm{~m}$ diameter profile, the LATR is the largest sub-Kelvin cryostat to be installed on a steerable telescope to date. The development of this instrument paves the way for future experiments that require large-volume ultracold cryostats such as CMB-S4 (Abazajian et al. 2016; Abitbol et al. 2017).

\section{LATR Design}

The SO LATR has six temperature stages: $300 \mathrm{~K}, 80 \mathrm{~K}$, $40 \mathrm{~K}, 4 \mathrm{~K}, 1 \mathrm{~K}$, and $100 \mathrm{mK}$. The design of the LATR vacuum shell is driven by the requirements of being able to withstand atmospheric pressure, the mechanical interfaces associated with mounting it in the LAT, and the desire to minimize the mass that the corotating LAT interface would need to support. Based on finite-element analysis (FEA) results, we have adopted a two-piece cylindrical shell design (Figure 3). Two aluminum ribs are welded to the back section cylinder for additional strength. The front plate converges to a design with 13 hexagonal openings, reinforced structures, and light-weighted pockets on a $6 \mathrm{~cm}$ thick aluminum plate (Figure 3 ).

The LATR cryogenic stages mass more than one metric ton. Mechanically supporting this mass with the required stability and optical alignment while minimizing the thermal conductance is a significant challenge. To solve this, we use thinwalled glass epoxy laminate (G-10CR) to mechanically support the $80 \mathrm{~K}, 40 \mathrm{~K}$, and $4 \mathrm{~K}$ stages. Throughout this paper, we use G-10 to refer exclusively to G-10CR. This design draws on the legacies of both AdvACT (Thornton et al. 2016) and SPIDER (Gudmundsson et al. 2015). AdvACT used a similar cylindrical G-10 design to mechanically support the $40 \mathrm{~K}$ and $4 \mathrm{~K}$ stages. However, for the LATR, the diameter of such a cylinder would be nearly $2.4 \mathrm{~m}$. When cooled from $300 \mathrm{~K}$ to $40 \mathrm{~K}$, the diameter of such a structure would contract by $2.5 \mathrm{~cm}$, resulting in an unacceptable radial stress on the G-10, and significantly weakening the structure. Inspired by SPIDER, we break up the cylinders by using individual G-10 tabs to support each cryogenic stage. As detailed in Table 2, the $80 \mathrm{~K}$ stage is supported by 12 G-10 tabs from the front of the vacuum shell; the $40 \mathrm{~K}$ stage is supported by $24 \mathrm{G}-10$ tabs from the middle of the vacuum shell; and the $4 \mathrm{~K}$ (and colder) stage is supported by another $24 \mathrm{G}-10$ tabs from the $40 \mathrm{~K}$ stage. The 300-40 K G-10 tabs can be seen in Figure 4. 
Table 1

The SO LAT Survey Specifications

\begin{tabular}{|c|c|c|c|c|c|c|}
\hline $\begin{array}{l}\text { Frequency } \\
(\mathrm{GHz})\end{array}$ & $\begin{array}{c}\text { FWHM } \\
\text { (arcminute) }\end{array}$ & $\begin{array}{c}\text { Baseline } \\
(\mu \mathrm{K}-\operatorname{arcmin})\end{array}$ & $\begin{array}{c}\text { Goal } \\
(\mu \mathrm{K}-\operatorname{arcmin})\end{array}$ & $\begin{array}{c}\text { Frequency } \\
\text { Bands }\end{array}$ & $\begin{array}{l}\text { Detector } \\
\text { Number }\end{array}$ & $\begin{array}{l}\text { Optics } \\
\text { Tubes }\end{array}$ \\
\hline 25 & $8.4^{*}$ & 71 & 52 & $\mathrm{LF}$ & 222 & 1 \\
\hline 39 & $5.4^{*}$ & 36 & 27 & & 222 & \\
\hline 93 & 2.0 & 8.0 & 5.8 & MF & 10,320 & 4 \\
\hline 145 & 1.2 & 10 & 6.3 & & 10,320 & \\
\hline 225 & 0.9 & 22 & 15 & UHF & 5160 & 2 \\
\hline
\end{tabular}

Note. The SO LAT projected survey sensitivity with $f_{\text {sky }}=0.4$. This represents the nominal configuration for the first deployment, with seven of the 13 OTs installed (see Section 3.3 for OT design). The quoted detector numbers assume three detector wafers per OT. With the use of dichroic pixels, the six targeted bands are split into three categories: low frequency (LF), medium frequency (MF), and ultrahigh frequency (UHF). The baseline sensitivity includes an additional uncertainty factor to account for approximated instrument systematic noise, while the goal sensitivity assumes the instrument noise is negligible. A detailed explanation of the value here can be found in The Simons Observatory Collaboration (2019).

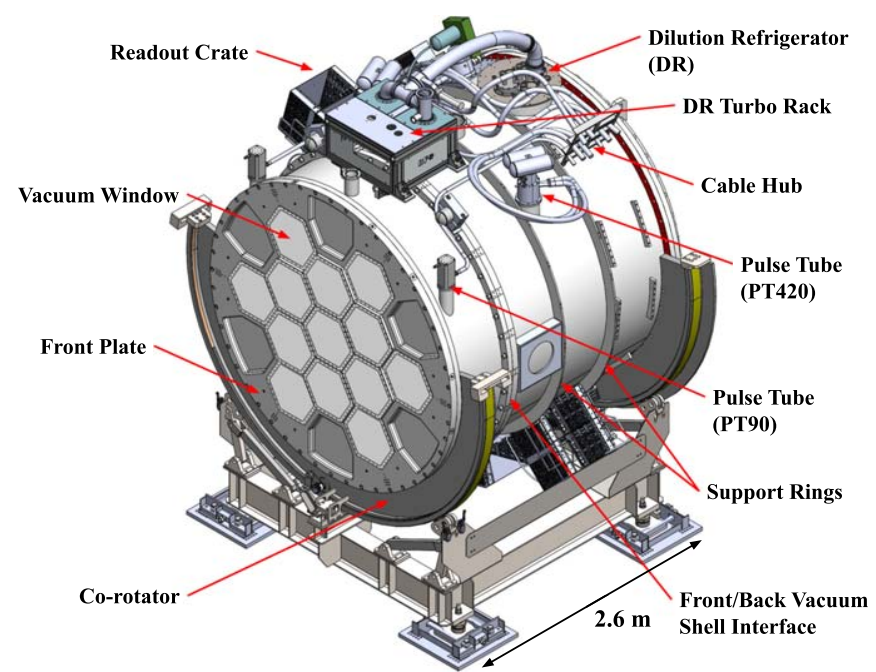

Figure 3. LATR external components are shown in this figure. The front plate with 13 hexagonal windows is labeled. The corotator at the bottom supports the LATR in the LAT (Figure 1), and allows the LATR to maintain its orientation with respect to the LAT mirrors when the telescope changes elevation. Two of the pulse tube coolers on one side are labeled, including a one-stage $80 \mathrm{~K}$ cooler (PT90) and a two-stage $40 \mathrm{~K} / 4 \mathrm{~K}$ cooler (PT420). The DR at the back of the LATR is labeled. Also labeled is one of the readout crates around the LATR; these crates house a number of critical electronics for detector data readout. All hoses and cables travel to the central hub (metal frame on the top right) on the cryostat before going to the cable wrap (Figure 1).

The detector arrays and cold optical components at $\leqslant 4 \mathrm{~K}$ (including lenses and filters) are packaged in OTs, which are attached directly to the $4 \mathrm{~K}$ cold plate with relatively easy access. The detailed design of the OT is discussed in Section 3.3. Mounting all optical components in the optics path within a single OT makes it simpler to precisely control the relative position of the optical components. The modular design also allows one to easily reconfigure the receiver by swapping out any individual optics tube without impacting the other tubes. The interior of each OT provides mechanical and thermal isolation for $4 \mathrm{~K}, 1 \mathrm{~K}$, and $100 \mathrm{mK}$ components. The $4 \mathrm{~K}$ OT stage is attached directly to the $4 \mathrm{~K}$ plate, and the $1 \mathrm{~K}$ and $100 \mathrm{mK}$ stages are cooled via $1 \mathrm{~K}$ and $100 \mathrm{mK}$ thermal Back-up Structures (BUSs). The $1 \mathrm{~K}$ and $100 \mathrm{mK}$ thermal BUSs are oxygen-free-high-conductivity (OFHC) copper web structures, which efficiently distribute the cooling power from
Table 2

G10 Tab Specifications

\begin{tabular}{lcccc}
\hline \hline Stage & $\begin{array}{c}\text { Width } \\
(\mathrm{mm})\end{array}$ & $\begin{array}{c}\text { Length } \\
(\mathrm{mm})\end{array}$ & $\begin{array}{c}\text { Number of } \\
\text { Tabs }\end{array}$ & $\begin{array}{c}\text { Armstrong A-12 } \\
\text { Glue Area }\left(\mathrm{cm}^{2}\right)\end{array}$ \\
\hline $300-80 \mathrm{~K}$ & 140 & 180 & 12 & 29 \\
$300-40 \mathrm{~K}$ & 160 & 150 & 24 & 36 \\
$40-4 \mathrm{~K}$ & 150 & 195 & 24 & 33 \\
\hline
\end{tabular}

Note. The geometry and number of tabs. All tabs are $2.4 \mathrm{~mm}$ thick with different width and length. The G-10 meets NIST G-10 CR process specification and conforms to MIL-I-247682 Type GEE/CR.

the dilution refrigerator (DR) to the back of the OTs (Figures 4 and 11).

The LATR design also incorporates 12 large radial feedthroughs penetrating the $300 \mathrm{~K}, 40 \mathrm{~K}$, and $4 \mathrm{~K}$ stages to accommodate the pulse tubes, the dilution refrigerator, and cable feedthroughs. The radial feedthroughs are designed to remain thermally coupled to the relevant heat shield while avoiding light leaks and allowing for differential motion between the heat shields and $300 \mathrm{~K}$ mounting plate. We also paid attention to ensuring the components could be easily installed and removed, in keeping with the modular design and future upgrade potential of the 13 optics tube configuration.

\subsection{Mechanical Design}

The SO LATR mechanical structure is shown in Figure 4. The vacuum shell, cold plates, and radiation shields were manufactured by Dynavac. ${ }^{30}$

The $300 \mathrm{~K}$ vacuum shell consists of the front plate, the front shell, the back shell, and the back plate; all constructed of aluminum 6061-T6. The size of the cryostat was chosen to be $2.4 \mathrm{~m}$ in diameter to accommodate 13 OTs and allow for mechanical supports and thermal isolation/shielding.

The front plate is a $6 \mathrm{~cm}$ thick flat plate with 13 densely packed hexagonal window cutouts to allow for maximum illumination of the detector arrays. Optimizing the front plate was one of the most challenging aspects of the LATR design. Optical and sensitivity requirements warrant maximizing open apertures via closely spaced windows. However, removing more

30 https://www.dynavac.com/ 


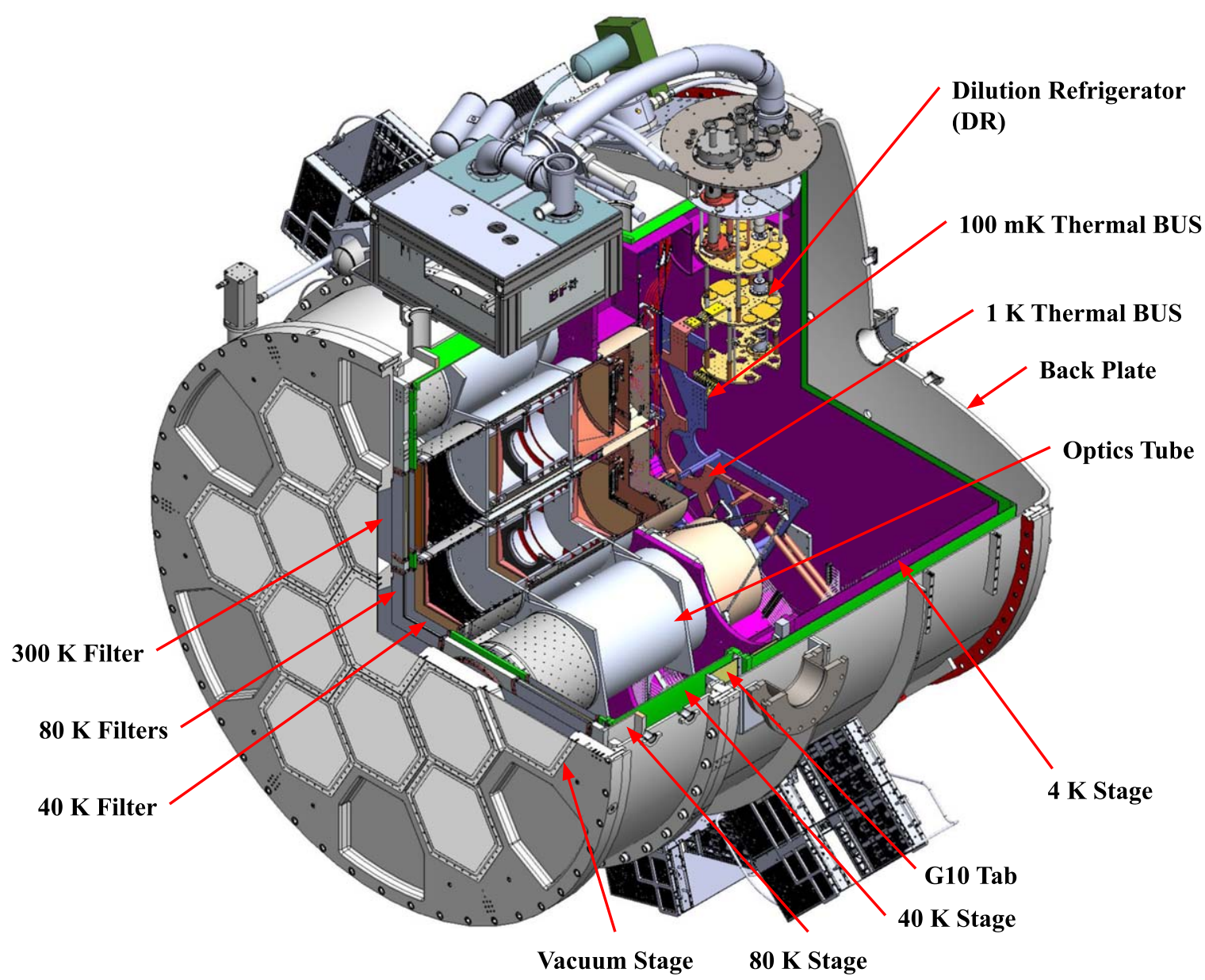

Figure 4. The LATR cut out to display the internal structures. From the front to the back, the LATR consists of the $300 \mathrm{~K}$ vacuum stage, $80 \mathrm{~K}$ stage (gray), $40 \mathrm{~K}$ stage (green), and $4 \mathrm{~K}$ stage (purple). Section 3 describes the design of each stage in detail. The $80 \mathrm{~K}$ stage is supported by $12 \mathrm{G}-10$ tabs from the front of the vacuum shell; the $40 \mathrm{~K}$ stage is supported by $24 \mathrm{G}-10$ tabs from the middle of the vacuum shell. One of the $300-40 \mathrm{~K} \mathrm{G}-10$ tabs is visible and labeled. Inside the $4 \mathrm{~K}$ cavity, the $1 \mathrm{~K}$ and $100 \mathrm{mK}$ stages distribute the cooling power from the dilution refrigerator to individual OTs (see Figure 11). Infrared filters on the cryostat are shown at the $300 \mathrm{~K}$, $80 \mathrm{~K}$, and $40 \mathrm{~K}$ stages in front of the OTs. The OTs contain the optical components at $\leqslant 4 \mathrm{~K}$, as well as the detector arrays. The OT design is discussed in Section 3.3 and its internal structure is displayed in Figure 9.

material drives the plate to be thicker, which eventually leads to a conflict between the diverging optical beam and the window spacing. Ultimately, the OT spacing was primarily driven by the optimization of the front plate design. The hexagonal windows are tapered to match the diverging beam, leaving as much material as possible in order to reduce the bending and stress on the front plate. Alternative designs and materials were considered, including machining the plate with a domed shape, either bowing in or out. These were rejected due to the complexity and expense of machining them. Additionally, doming the front plate would stagger the windows axially; this would require all further optical elements to be axially staggered to maintain consistency of the optical chain, greatly complicating the design of the cold optics.

A key mechanical challenge for the vacuum shell was managing the level of bending of the comparatively narrow struts around the 13 hexagonal optic tube openings in the front plate under atmospheric pressure. After consultation with an external engineering firm (PVEng ${ }^{31}$ ), we addressed this concern by increasing the thickness of the front vacuum shell wall and adding stiffening ribs. Overall, the minimum factor of safety (FoS) on the vacuum shell is $>3$ at 1 atmosphere of

$\overline{31}$ Pressure Vessel Engineering Limited, https://www.pveng.com/. pressure, with the lowest FoS on the front plate. Figure 5 shows the expected deformation of the final front plate design from FEA, magnified by a factor of 10 . These results are for sealevel atmospheric pressure. While the pressure at the highelevation site in Chile is only $\sim 0.5$ bar, the integration testing of the LATR is being done at lower elevations.

Sensitivity requirements drive the windows to be as thin as possible, which has to be balanced against the need to withstand atmospheric pressure. The LATR utilizes $1 / 8$ inch thick hexagonal windows made of antireflection (AR) coated ultra-high-molecular-weight polyethylene. Each hexagonal window has its own O-ring. A single window has been vacuum tested under atmospheric pressure for more than 36 months with no indication of reduced structural performance or leakage. The LATR has been tested with seven $1 / 8$ inch thick windows. However, the majority of the in-lab (sea-level) testing has been performed with $1 / 4$ inch thick windows, to remove the risk of a catastrophic failure event that might damage other parts of the receiver. One double-sided IR blocking filter fabricated by Cardiff University (Ade et al. 2006; Section 7) is mounted on the back of the front plate behind each window, to reduce the optical loading entering the cryostat. The cold optics design is discussed in detail in Section 3.3.1. 

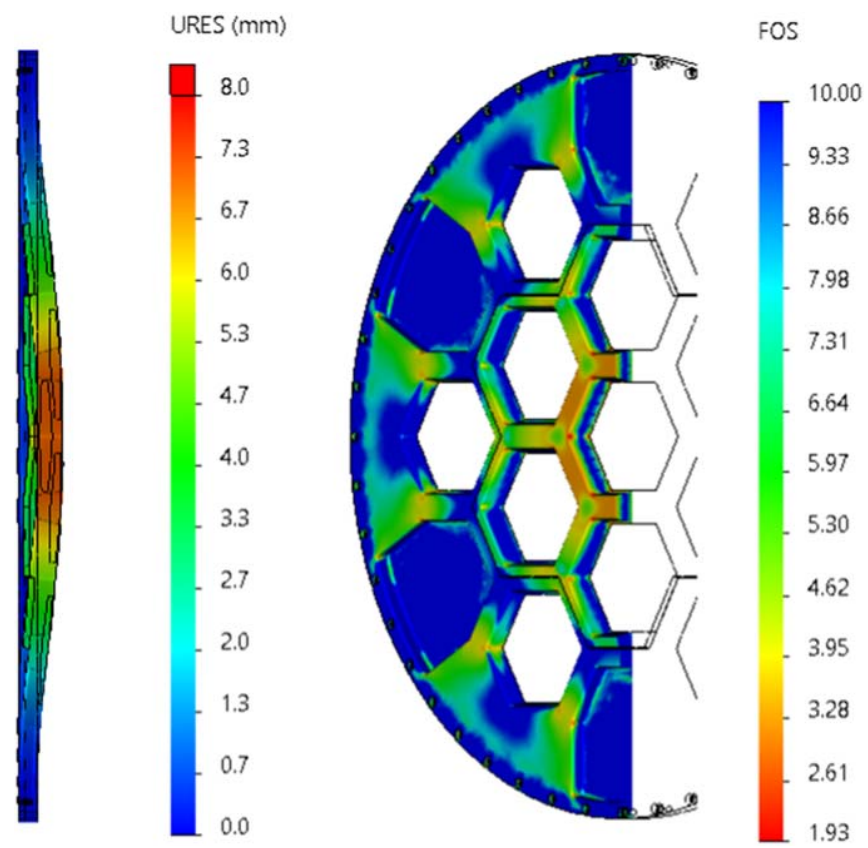

Figure 5. Left: Resultant displacement (URES) plot showing the bowing of the front plate under 1 atmosphere of pressure. The displacement scale is exaggerated 10 times. Right: FoS plot for the vacuum shell under one atmospheric pressure at sea level. The minimum FoS is in the corners of the center window and is due to unphysical stress concentrations from the finite size of the mesh. The actual FoS on the surface of the vessel is $>3$.

The $80 \mathrm{~K}$ stage consists of a $2.1 \mathrm{~m}$ diameter, $2.54 \mathrm{~cm}$ thick circular plate made of aluminum 1100-H14 and a short $80 \mathrm{~K}$ shield made of aluminum 6061-T6. It is designed to intercept most of the optical loading entering the cryostat, reducing the load on the $40 \mathrm{~K}$ stage. The 1100 series aluminum was used due to its higher thermal conductivity. The $80 \mathrm{~K}$ plate contains one double-sided IR blocking filter and one AR-coated alumina filter for each OT (Golec et al. 2020). The alumina filters act as an IR absorber as well as a prism to bend the off-axis beams back parallel to the long axis of the cryostat (Dicker et al. 2018). The outside of the $80 \mathrm{~K}$ stage is covered with 30 layers of multilayer insulation (MLI) ${ }^{32}$ to reduce the blackbody radiative load coming from the $300 \mathrm{~K}$ shell. The structural support of the $80 \mathrm{~K}$ stage is located at the front of the vacuum shell. Thus, the entire $80 \mathrm{~K}$ stage can be installed or disassembled independently from the rest of the cryostat.

The $40 \mathrm{~K}$ stage consists of two cylinders made of 1100 aluminum and two plates and a ring made of 6061-T6 aluminum. It is suspended directly from the $300 \mathrm{~K}$ vacuum shell, located on the vacuum shell back section (Figure 4). This provides a natural break in the vacuum shell for ease of assembly. There is a thick structural flange on either side of this break that minimizes stress on the vacuum shell itself (Figure 3). Like the $80 \mathrm{~K}$ stage, the $40 \mathrm{~K}$ stage is supported by G-10 tabs. Again, we did a thorough FEA validation to verify the structural strength of the G-10 tab support (OrlowskiScherer et al. 2018). To reduce loading on the $4 \mathrm{~K}$ stage, the $12.7 \mathrm{~mm} 40 \mathrm{~K}$ filter plate at the front contains a third IR blocking filter for each OT. We covered the entirety of the $40 \mathrm{~K}$ assembly with 30 layers of MLI and wrapped each of the G-10 tabs in 20 layers to reduce $300 \mathrm{~K}$ radiation loading. To vent the

\footnotetext{
${ }^{32}$ RUAG Holding AG, https://www.ruag.com/en/.
}

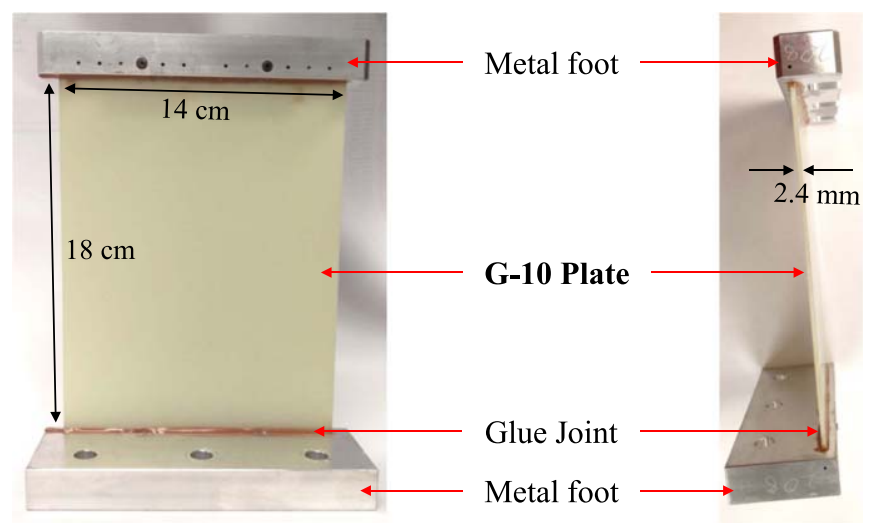

Figure 6. An example of a 300-80 K G-10 tab is shown from two perspectives. Two aluminum "feet" are glued to G10 plate as mechanical interfaces. Armstrong A-12 was chosen as the glue because of its mechanical and cryogenic performance. Each G-10 tab is serialized as shown in the side-view photo on the right.

inside of the $40 \mathrm{~K}$ cavity, evacuation blocks mounted on the back lid of the $40 \mathrm{~K}$ shell were designed to allow air to escape without adding an easy path for light leaks.

The $4 \mathrm{~K}$ stage consists of a $2.08 \mathrm{~m}$ diameter, $2.54 \mathrm{~cm}$ thick circular plate, a $4 \mathrm{~K}$ radiation shield, and a thin $4 \mathrm{~K}$ back plate, all fabricated from 6061 aluminum. The main $4 \mathrm{~K}$ plate is significantly thicker than the plates for the $40 \mathrm{~K}$ stage because this structure supports the OTs (Section 3.3). The $4 \mathrm{~K}$ radiation shield has recesses to allow the pulse tube thermal connections to be made without penetrating the $4 \mathrm{~K}$ cavity (Figure 11). These cutouts allow us space to make thermal contact over a significant area while minimizing the risk of light leaks into the $4 \mathrm{~K}$ cavity, where the $1 \mathrm{~K}$ and the $100 \mathrm{mK}$ components reside.

Due to their importance in reducing parasitic thermal load, supporting the mass of the cold stages of the cryostat, and setting the reference location for the $40 \mathrm{~K}$ and $4 \mathrm{~K}$ stages, the G-10 tabs were the subject of significant design focus. The tabs consist of two "feet" connected by a flat sheet of G-10, glued together in a precision jig using Armstrong A-12 epoxy. An example of such a tab is shown in Figure 6. The tabs can flex radially, allowing them to accommodate the high differential thermal contraction (on the order of $2.5 \mathrm{~cm}$ in diameter) between the cold stages and the vacuum shell during cooling. Extensive FEA was performed to simulate the structural strength of the G-10 tabs (Orlowski-Scherer et al. 2018), which determined that the factor of safety for the structural components of the tabs is $>8$, excluding the effects of bonding the G-10 tab to the aluminum foot. A pull test of an assembled tab was performed to determine the strength of the glue bond; it failed via deadhesion from the aluminum surface at $21 \mathrm{kN}$, implying a glue adhesive strength of $590 \mathrm{MPa}$. While this force was lower than predicted from the manufacturer listed bond strength, the resulting FoS of 6 still satisfies our requirements. The geometry and number of tabs are given in Table 2 . The G-10 was supplied by Professional Plastics. ${ }^{33}$

To increase the sensitivity of the TES detectors, all detector components and readout chips are cooled to below $100 \mathrm{mK}$ (Mather 1984) with a Model LD400 DR manufactured by Bluefors. ${ }^{34}$ To reduce thermal gradients on the $1 \mathrm{~K}$ and $100 \mathrm{mK}$ stages, we implement two thermal BUSs comprised of two

\footnotetext{
33 https://www.professionalplastics.com/

34 https://www.bluefors.com/
} 
Table 3

LATR Thermal Loading Estimates

\begin{tabular}{lccccccccc}
\hline \hline $\begin{array}{l}\text { Stage } \\
(\mathrm{K})\end{array}$ & $\begin{array}{c}\text { Support } \\
(\mathrm{W})\end{array}$ & $\begin{array}{c}\text { Cabling } \\
(\mathrm{W})\end{array}$ & $\begin{array}{c}\text { Radiative } \\
(\mathrm{W})\end{array}$ & $\begin{array}{c}\text { Optical } \\
(\mathrm{W})\end{array}$ & $\begin{array}{c}\text { LNAs } \\
(\mathrm{W})\end{array}$ & $\begin{array}{c}\text { Attenuation } \\
(\mathrm{W})\end{array}$ & $\begin{array}{c}\text { Arrays } \\
(\mathrm{W})\end{array}$ & $\begin{array}{c}\text { Total } \\
(\mathrm{W})\end{array}$ & $\begin{array}{c}\text { Available Power } \\
(\mathrm{W})\end{array}$ \\
\hline 80 & 3.17 & 0.0261 & 6.59 & 50.7 & $\mathrm{~N} / \mathrm{A}$ & $\mathrm{N} / \mathrm{A}$ & $\mathrm{N} / \mathrm{A}$ & 60.5 & 180 \\
40 & 9.51 & 14.7 & 25.5 & 0.0256 & 5.80 & 0.00958 & $\mathrm{~N} / \mathrm{A}$ & 55.5 & 110 \\
4 & 0.313 & 0.776 & 0.197 & 0.359 & 0.576 & 0.00157 & $\mathrm{~N} / \mathrm{A}$ & 2.22 & 4.00 \\
1 & $2.04 \times 10^{-3}$ & $0.326 \times 10^{-3}$ & $5.47 \times 10^{-6}$ & $0.378 \times 10^{-3}$ & $\mathrm{~N} / \mathrm{A}$ & $0.111 \times 10^{-3}$ & $\mathrm{~N} / \mathrm{A}$ & $2.85 \times 10^{-3}$ & $25.0 \times 10^{-3}$ \\
0.1 & $43.6 \times 10^{-6}$ & $8.33 \times 10^{-6}$ & $0.405 \times 10^{-6}$ & $4.06 \times 10^{-6}$ & $\mathrm{~N} / \mathrm{A}$ & $\mathrm{N} / \mathrm{A}$ & $10.1 \times 10^{-6}$ & $66.5 \times 10^{-6}$ & $500 \times 10^{-6}$ \\
\hline
\end{tabular}

Note. Loading estimates for each temperature stage of the LATR split by source. The provided load estimates are for 13 OTs. The cooling power at $80 \mathrm{~K}$ is supplied by two PT 90 coolers, the power at $40 \mathrm{~K}$ and $4 \mathrm{~K}$ is supplied by two PT420 coolers, the power at $1 \mathrm{~K}$ by the DR still stage, and the $100 \mathrm{mK}$ by the DR mixing chamber stage.

wheel-like structures made of OFHC copper (Figures 4 and 11). Neither of the BUSs are gold-plated, due to their complexity and the cost involved. Thermal FEA shows that the temperature gradient across both thermal BUSs should be $\sim 5 \mathrm{mK}$, which satisfies our gradient requirements of $\lesssim 10 \mathrm{mK}$. During cryogenic validation, the actual temperature gradient measured on the $100 \mathrm{mK}$ BUS is $\lesssim 10 \mathrm{mK}$, higher than the FEA value but still below the requirement. Based on calculated loading from conduction and measured total loading, the inferred radiative load on the $100 \mathrm{mK}$ thermal BUS is $\ll 1 \mu \mathrm{W}$. We thus conclude that the gold plating is not necessary for the BUSs. Detailed cryogenic measurements are discussed in Section 4.2. From the thermal BUS cold finger, individual gold-plated copper straps, manufactured by TAI, ${ }^{35}$ extend down to attach to the $1 \mathrm{~K}$ and $100 \mathrm{mK}$ cold fingers inside each OT. The $1 \mathrm{~K}$ thermal BUS is supported from the $4 \mathrm{~K}$ plate with carbon fiber tripods, while the $100 \mathrm{mK}$ thermal BUS is supported from the $1 \mathrm{~K}$ thermal BUS via carbon fiber trusses. We chose the twill-ply carbon fiber tubing for these legs as we find that the unidirectional-ply carbon fiber has a tendency to splinter. The carbon fiber used for these applications was sourced from Clearwater Composites. ${ }^{36}$

\subsection{Cryogenic Design}

In order to reach the desired detector sensitivity, the LATR cryogenic system must maintain all 62,000 detectors at a stable temperature below $100 \mathrm{mK}$ while minimizing the in-band optical loading onto the detectors. The system must also be mechanically rigid, with required tolerances on optical alignment of the elements that are fractions of a millimeter over the $2.4 \mathrm{~m} \times 2.6 \mathrm{~m}$ scale of the system. Given that the LATR needs to cool $1200 \mathrm{~kg}$ of material to $4 \mathrm{~K}$ and $200 \mathrm{~kg}$ to below $100 \mathrm{mK}$, it is challenging to design the cryogenic system to cool down the internal structures effectively. The $80 \mathrm{~K}$ stage uses two PT90 pulse tubes, while the $40 \mathrm{~K}$ and $4 \mathrm{~K}$ stages are cooled by two PT420 pulse tubes supplied by Cryomech. ${ }^{37}$ The $1 \mathrm{~K}$ and $100 \mathrm{mK}$ stages are cooled by the DR, which is backed by an additional PT420 pulse tube, supplementing the cooling power of the main cryostat. As a design contingency, the LATR can accommodate an additional PT90 and PT420 pulse tube cooler for the $80 \mathrm{~K}$ and $40 \mathrm{~K} / 4 \mathrm{~K}$ stages, respectively. However, these additional coolers are not required based on our cryogenic validation tests.

Managing and minimizing the thermal loads to meet the cryogenic performance specifications drives almost every

\footnotetext{
$\overline{35}$ Technology Applications, Inc. (TAI), https://www.techapps.com/.

36 https://www.clearwatercomposites.com/

37 https://www.cryomech.com
}

aspect of the thermal design. The base temperature of the pulse tube is a function of the total thermal load on the system. Thermal load on each stage includes conductive load from supporting structures, radiative load from the warmer stages and incoming light, conductive load from cables, and cryogenic electrical components. Estimates of all of these were included in thermal models, summarized in Table 3.

The large $2.4 \mathrm{~m} \times 2.6 \mathrm{~m}$ scale of the receiver poses its own challenges to the cryogenic design. Reaching the target temperatures at the cold head of the pulse tube coolers or dilution refrigerator is a necessary but not sufficient condition. The thermal design must also include sufficient thermal conductivity within each temperature stage to keep the thermal gradient below the required level. For example, the farthest point on the $40 \mathrm{~K}$ filter plate is $\sim 2 \mathrm{~m}$ from the nearest pulse tube with five mechanical joints in the thermal path (Figure 4). If the thermal link is weak, a small amount of power can lead to a large temperature gradient along the path, resulting in an elevated optical filter temperature, without substantively affecting the pulse tube base temperature. In most cases, the thermal path follows the mechanical structure. However, there are exceptions. For example, 6061 aluminum is used for the mechanical structure of the $4 \mathrm{~K}$ cold plate to meet the optical alignment requirements, and supplemented by the softer but more conductive 1100 aluminum to meet the thermal requirements. In another case, flexible thermal strips connect the coolers and cryogenic stages to transfer heat while allowing for the differential contraction between the two.

Finally, it is important to minimize temperature gradient during cooldown. Based on the mass of the cold structure, bringing the system from $300 \mathrm{~K}$ to the target temperature requires removing 350 million joules of energy from the system. Gradients are important because the pulse tube cooling efficiency is a very steep function of the cold head temperature. The PT420 second stage ( $4 \mathrm{~K}$ cold head) can remove up to $225 \mathrm{~W}$ when it is at $300 \mathrm{~K}$, but only $2 \mathrm{~W}$ when it is cooled to $4 \mathrm{~K}$. Therefore, the most efficient use of the pulse tube during cooling is achieved when thermal gradients are minimized across the cryostat, while pulse tube cold head temperature is maximized. A large temperature gradient between the cold head and stage means the pulse tube temperature is well below the rest of the stage, and thus the pulse tube can remove less power than it would if the entire system was at the same temperature. A model of the material properties as a function of temperature is included in the design to ensure sufficient thermal conduction throughout the relevant parts of the LATR during cooldown. The cooldown predictions are shown in 
Section 3.2.2, and the measured cooldown times are presented in Section 4.2.

\subsubsection{Thermal Modeling}

Thermal modeling included a careful accounting of all sources of loading at each stage. To compute the thermal loading from supports, we first compiled a library of materials and their thermal conductivities at cryogenic temperatures. We obtained measurements from the National Institute for Standards and Technology (NIST) cryogenic material properties database (Marquardt et al. 2002; NIST 2009) and from Adam Woodcraft's low-temperature material database (Woodcraft \& Gray 2009). The total conductive load for a support is the integrated thermal conductivity between the temperatures on the high and low ends. The conductivity of the cables were computed in the same way, with the conductivity of the coax cables provided by Coax Co. ${ }^{38}$

The thermal conduction due to radiation on surfaces covered in MLI was computed using the Lockhead equation (Keller et al. 1974):

$$
\begin{aligned}
q_{\mathrm{tot}}= & \frac{C_{\mathrm{c}} N^{2.56} T_{\mathrm{m}}}{n}\left(T_{\mathrm{h}}-T_{\mathrm{c}}\right) \\
& +\frac{C_{\mathrm{r}} \epsilon_{0}}{n}\left(T_{\mathrm{h}}^{4.67}-T_{\mathrm{c}}^{4.67}\right),
\end{aligned}
$$

which accounts for both the radiative load between layers of the MLI blanket (first term) and the conductive loading through the layers of the blanket (second term). In Equation (1), $q_{\text {tot }}$ is the total thermal load on the MLI, $C_{\mathrm{c}}=8.95 \times 10^{-5}$ is a numerical constant defining the MLI conductive heat transfer, $N$ is the MLI layer density in layers per centimeter, $T_{\mathrm{m}}$ is the mean MLI temperature, taken to be $T_{\mathrm{m}}=\frac{T_{\mathrm{h}}-T_{\mathrm{c}}}{2}, n$ is the number of MLI layers, $T_{\mathrm{h}}$ is the hot-side temperature in Kelvin, $T_{\mathrm{c}}$ is the coldside temperature in Kelvin, $C_{\mathrm{r}}=5.39 \times 10^{-7}$ is a numerical constant defining the MLI radiative heat transfer, and $\epsilon_{0}=0.031$ is the MLI emissivity (Keller et al. 1974).

To calculate the radiative and optical loading throughout the LATR, we created a custom Python package that models the thermal performance of all filter elements in an ideal radiative environment. The code estimates the total power emitted and absorbed at each temperature stage by performing a radiative transfer calculation using numerical ray optics for individual optics tubes. The circularly symmetrized geometry, the spectral properties of the filters, and the emissivity of temperature stage walls are used as inputs to this model. An additional output of this simulation is a set of radial temperature profiles for all filter elements (computed using temperature-dependent thermal conductivities of the filter materials). The resultant thermal loads for each stage are shown in Table 3.

Power dissipation from electrical components such as the detector readout low-noise amplifiers (LNAs) and TES biasing was also included in the thermal model. The LNA power dissipation was computed by multiplying the LNA drain current by its drain voltage, using the nominal current and voltage from the specification sheet provided by Low Noise Factory. ${ }^{39}$

We used the COMSOL ${ }^{40}$ software suite to estimate thermal gradients across our $80 \mathrm{~K}, 40 \mathrm{~K}, 4 \mathrm{~K}, 1 \mathrm{~K}$, and $100 \mathrm{mK}$ stages.

\footnotetext{
38 http://www.coax.co.jp/en/

39 https://www.lownoisefactory.com/

40 https://www.comsol.com
}

Our simulations used the computer-aided design (CAD) model of the relevant thermal stage, with some simplifications that only minimally impact the accuracy of the simulations-for example, suppressing screw holes. Using the material library we developed for the thermal model, we applied a material to each part, specifying its thermal conductivity as a function of temperature. Thermal loads were distributed throughout the model. To include the effect of the relevant cryocooler, a measured load curve of that cryocooler as a temperaturedependent negative heat flux is applied to the cold head of the cryocooler. These simulations allowed us to identify which areas were cryogenically critical, leading us to make those areas thicker and more conductive. The simulations showed that, to reduce the gradients throughout the stages, the radiation shields play a crucial role in conducting the cooling power besides shielding radiation. As a result of this, for the $40 \mathrm{~K}$ stage that relies heavily on the shield to cool the filter plate, we used A11100, the thermal conductivity of which is significantly higher than that of Al6061. A summary of our predicted gradients and the LATR performance during validation and testing can be found in Section 4.2.

During the lab-testing phase, we performed additional simulations in conjunction with our cryogenic validation. For these simulations, we would mimic a given validation test setup, including whichever components were installed and using the measured cryocooler load curves. We would then compare the gradients observed in the simulations to those observed in our validation testing. One major difficulty in the thermal validation of our cryostat at all temperature stages was that we were only able to put a limited number of thermometers on each stage, making it difficult for us to identify the source of unaccounted-for loading. This, combined with the very long turnaround time of our cryostat, meant that we risked spending upward of a month tracking down heat leaks. Given a proposed load, we would add that load in the simulation and compare the resulting temperature gradients to those observed. While the result of the simulation is not precise, missing among other things contact resistance, it is accurate enough to provide a sanity check. An example simulation is shown in Figure 7.

\subsubsection{Cooldown Simulations}

With a cryostat the size of the LATR, the main challenge for a relatively fast cooldown is distributing the available cooling power and minimizing the gradients during the cooldown. At room temperature, the PT420s each provide up to $450 \mathrm{~W}$ of cooling power for the first stage and $225 \mathrm{~W}$ for the second stage. If all of the cryogenic material in the LATR were isothermal to the pulse tube heads, the cooldown time would be 5.3 days. However, gradients across the temperature stages reduce the pulse tube heads' temperatures, and hence their cooling power, such that a realistic simulation is required to estimate the true cooldown time.

We developed a code based on a finite difference method as described in Coppi et al. (2018). The results for the LATR with 13 OTs are presented in Figure 8. The warmer stages- $80 \mathrm{~K}$, $40 \mathrm{~K}$, and $4 \mathrm{~K}$ - cool relatively quickly, in less than 15 days. However, initial simulations showed that it could take up to 28 days for the coldest stages- $1 \mathrm{~K}$ and $100 \mathrm{mK}$ - to reach $4 \mathrm{~K}{ }^{41}$

\footnotetext{
${ }^{41}$ Mixture condensation, the process in which the DR reaches its base temperature of $100 \mathrm{mK}$, proceeds in a few hours once the DR has reached $4 \mathrm{~K}$.
} 


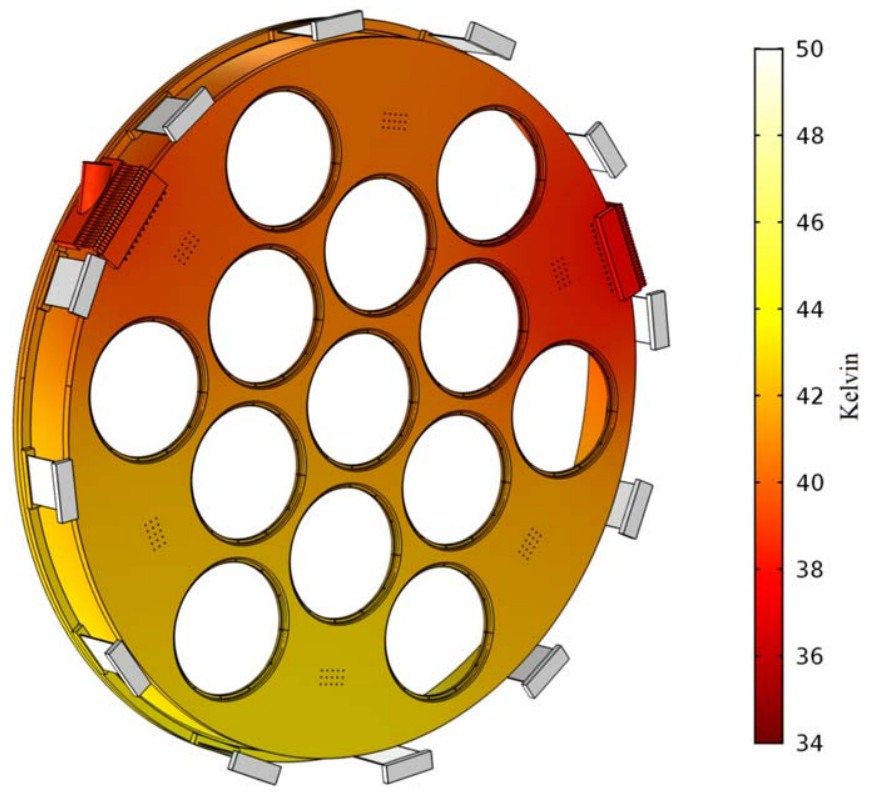

Figure 7. A thermal simulation of the $80 \mathrm{~K}$ stage. This simulation uses the individually calibrated PT90 load curves. It was performed to emulate a dark thermal validation run with the windows closed, hence there is no optical load applied. The conductive load through the G10 tabs was included, as is radiative heating from the $300 \mathrm{~K}$ stage. The color map shows the distribution of the thermal gradient with the lowest temperature around the two PT90 thermal straps and the highest temperature at the bottom of the plate. This is one example of the thermal simulations we conducted for all the temperature stages. The simulation as shown indicated that the $80 \mathrm{~K}$ stage would have $\sim 10 \mathrm{~K}$ gradient and be under $50 \mathrm{~K}$ if the total loading meets the estimations. The temperature gradient and loading at the $80 \mathrm{~K}$ stage measured during cryogenic validation test is discussed in Section 4.2.

A detailed cooldown time measurement with 1 OT and 3 filter sets is presented in Section 4.2.

To reduce cooldown time, we installed a nitrogen heat pipe and two mechanical heat switches. We studied multiple options for accelerating the cooldown process, such as using an external cooler to force cold gas circulation in an internal vacuum chamber (Alduino et al. 2019), liquid nitrogen flow through a plumbing system (Lizon \& Accardo 2010), nitrogen heat pipes connecting the $4 \mathrm{~K}$ stage to the colder stages (Duband et al. 2018), and the use of mechanical heat switches from the $4 \mathrm{~K}$ stage to the colder stages. The first two options were discarded due to mechanical design complications and the risk of developing large temperature gradients in the cryostat that would induce thermal stresses on critical components such as lenses and filters. Moreover, as stated above, the LATR has sufficient cooling power, but requires optimization of its distribution. We chose to apply the remaining two options because they are relatively easy to implement from a mechanical design point of view. Nitrogen heat pipes are intrinsically passive elements with high thermal conductivity between $63 \mathrm{~K}$ and $110 \mathrm{~K}$ and close to zero outside this range. A nitrogen heat pipe was custom installed on the DR by Bluefors. Mechanical heat switches have very high thermal conductivity when closed and zero when open, but require some activation mechanism. Mechanical heat switches manufactured by Entropy ${ }^{42}$ were chosen for the LATR. These heat switches have a nominal conductance of $0.5 \mathrm{~W} \mathrm{~K}^{-1}$ at $4 \mathrm{~K}$ when closed. Figure 11 shows a photo of the heat switches installed on the $4 \mathrm{~K}$ plate. One switch connects the $4 \mathrm{~K}$ and $1 \mathrm{~K}$ stages while

\footnotetext{
42 http://www.entropy-cryogenics.com/
}

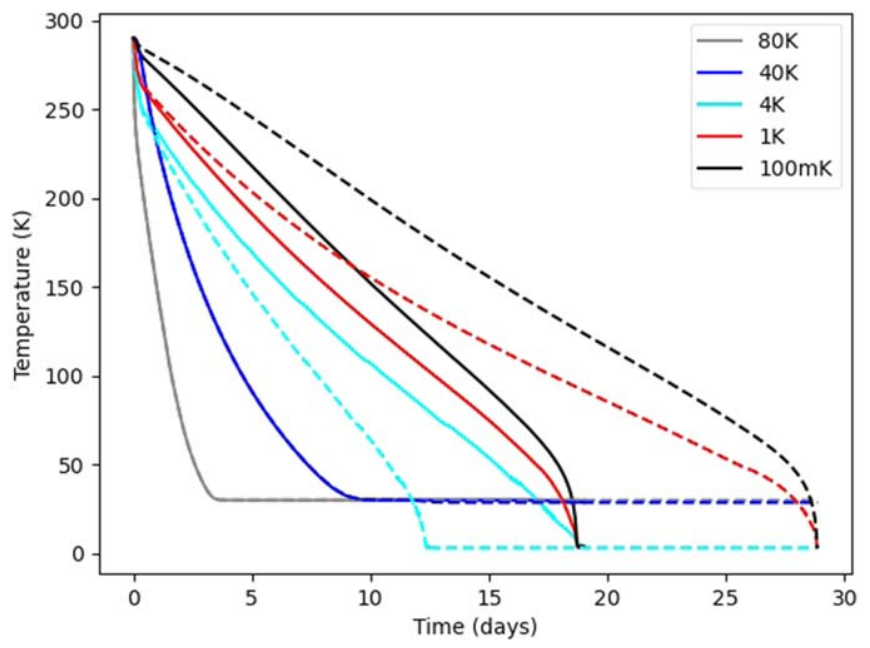

Figure 8. Simulated cooldown curve for a fully populated LATR with 13 OTs, showing each temperature stage. The solid lines show the result with mechanical heat switches connecting the $4 \mathrm{~K}$ stage with the $1 \mathrm{~K}$ and $100 \mathrm{mK}$ stages, while the dashed lines show the result without heat switches. The heat switches significantly reduce expected cooling time.

the other connects the $4 \mathrm{~K}$ and $100 \mathrm{mK}$ stages. With these heat switches, a fully equipped LATR (with 13 OTs) would cool in around 18 days according to our simulations. The actual cooldown time with one OT is discussed in Section 4.2.

\subsection{Optics Tubes}

The cryostat can accommodate a total of 13 optical chains, each with a dedicated set of detectors (see Table 1). Each optical chain consists of elements that are mounted on either the cryostat cold plates $(300 \mathrm{~K}, 80 \mathrm{~K}$, and $40 \mathrm{~K})$, or for the colder stages, in a large, self-contained OT (Figure 9) that has components at $4 \mathrm{~K}, 1 \mathrm{~K}$, and $100 \mathrm{mK}$. Each tube is roughly $40 \mathrm{~cm}$ in diameter and $130 \mathrm{~cm}$ long and is designed to be removable as a single unit from the rear of the cryostat. As detailed in Table 1, the initial seven optics tubes include one in the LF bands, four in the MF bands, and two in the UHF bands. A cross section of an MF tube is shown in Figure 9. The field of view for each OT is 1.3 in diameter, which is only partially filled by the three detector wafers.

\subsubsection{Cold Optics}

We selected a refractive cold optics design for its compactness. Inside each OT, three silicon lenses reimage the telescope focal plane onto a set of three hexagonal detector arrays (Dicker et al. 2018). Silicon is selected as the lens material due to its low loss and the outstanding performance of developed metamaterial AR coatings (Datta et al. 2013; Coughlin et al. 2018; Golec et al. 2020). The upper edge of each frequency channel is set by a set of low-pass edge (LPE) filters, which use a capacitive mesh design (Ade et al. 2006) to set a range of cutoff frequencies (e.g., $12.5 \mathrm{~cm}^{-1}$ to $6.2 \mathrm{~cm}^{-1}$ for MF). The cutoffs are also chosen to suppress out-of-band leaks from other filters. The LPE filters are complemented by infrared blocking filters at warmer stages (Tucker \& Ade 2006) to reduce the thermal loads on the colder stages. The designs implemented for the SO incorporate two metal mesh patterns printed onto each face of a polypropylene substrate to form double-sided IR blockers. The capacitive square copper patterns have a $15 \mu \mathrm{m}$ period; the polypropylene substrate is 


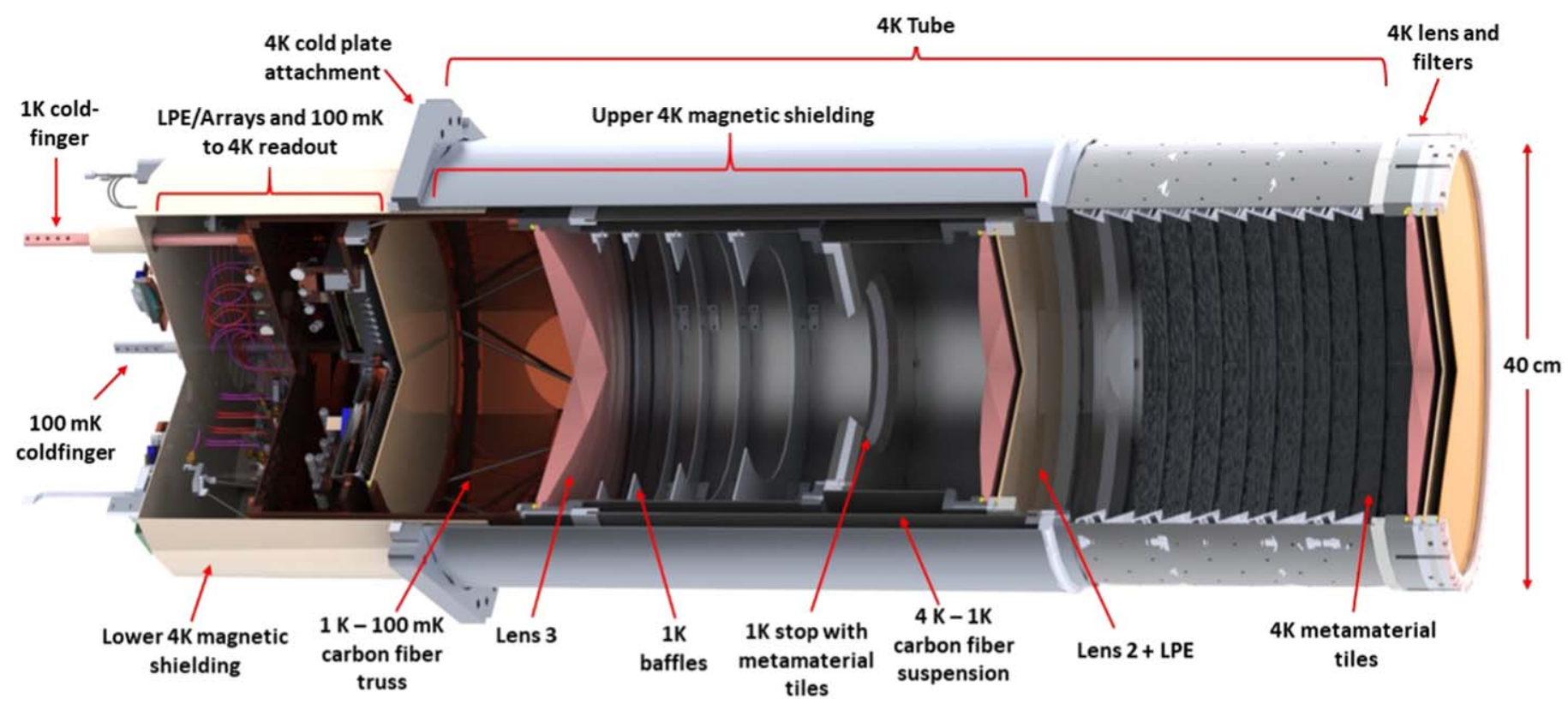

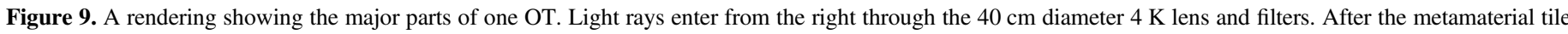

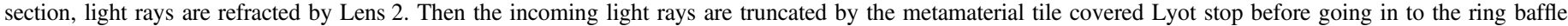

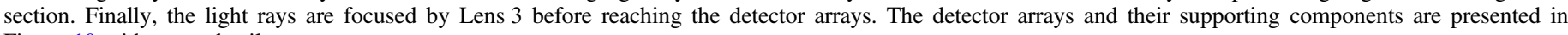
Figure 10 with more details.

thin $(4 \mu \mathrm{m})$ and absorbs very little of the in-band radiation. Thus, each device acts as a basic low-pass filter that is optimized for high reflectivity of near-IR radiation with virtually no attenuation of the science bands. Successive filters are placed between $300 \mathrm{~K}$ and $4 \mathrm{~K}$, to reflect the IR radiation emitted by these stages and to protect the thermal environment at the $1 \mathrm{~K}$ and $0.1 \mathrm{~K}$ stages. Combined, the optical elements consist of: a $3.1 \mathrm{~mm}$ thick AR coated window and an IR blocking filter at $300 \mathrm{~K}$; an IR blocking filter and alumina absorbing filter at $80 \mathrm{~K}$; an IR blocking filter at $40 \mathrm{~K}$; an IR blocking filter, LPE filter, and the first lens at $4 \mathrm{~K}$; an LPE filter, the second lens, the Lyot stop, and third lens at $1 \mathrm{~K}$; and the final LPE filter and detector arrays at $100 \mathrm{mK}$. See Figure 9 for the relative locations of optical elements inside the OT.

In this optics tube design, it is worth highlighting part of the $80 \mathrm{~K}$ design that makes it possible. The alumina filter at $80 \mathrm{~K}$ is wedge-shaped (except for the central OT) so that it acts as a prism in addition to acting as an IR blocking filter. This prism allows all OTs to be coaxial with the cryostat, which allows for a much more efficient use of space and greatly simplifies the installation/removal of the OTs. Each alumina filter has a notch machined into it for setting the proper angular orientation. We are pursuing several parallel AR coating techniques for this element to optimize cost versus performance. The baseline is to use metamaterial coatings on alumina (Golec et al. 2020).

The close-packed OTs prevent the use of traditional light baffling between $4 \mathrm{~K}$ filter and Lens 2 , since there is little space between the walls of the upper ( $4 \mathrm{~K}$ ) OT and the incoming beam. Since stray light is a major concern, significant effort was dedicated toward fabricating novel, low-profile metamaterial microwave absorbers (Gudmundsson et al. 2021; Xu et al. 2021). The goal was a design that had maximum absorptivity and minimum reflection, was capable of cooling down to cryogenic temperatures while maintaining mechanical integrity, and was relatively easy to install. The solution was injection-molded, carbon-loaded plastic tiles that create a gradient index AR coating (Xu et al. 2021). About 240 holes were laser-cut into the upper tube, allowing each tile to be screwed into place. A flat version of the tile was designed to attach to both sides of the $1 \mathrm{~K}$ Lyot stop. The region in between the stop and Lens 3 was baffled with standard ring baffles covered with a mixture of Stycast 2850 FT, coarse carbon powder, and fine carbon powder, because it has more radial clearance and is less critical for stray light. The $1 \mathrm{~K}$ radiation shield surrounding the detector arrays was blackened in the same manner.

\subsubsection{Mechanical Support Structures}

The windows and all filters are mounted using aluminum clamps (except for the $100 \mathrm{mK}$ LPE filter, which uses a copper clamp). The LPE filter clamps are axially spring-loaded to minimize the possibility of delamination due to the shear forces involved during large (radial) differential thermal contraction ( $\sim 6 \mathrm{~mm}$ ) between the aluminum mounts and the polypropylene filters. The spring is a commercial spiral beryllium copper structure. ${ }^{43}$ The lens mounts contain both an axial spring and a radial spring. The axial spring ensures firm thermal contact between the lens and mount without risking cracking the brittle silicon. The radial spring ensures the lens is centered at operating temperature by pushing the lens against two opposing hard points.

Each OT consists of a large $4 \mathrm{~K}$ cylindrical structure containing the $1 \mathrm{~K}$ and $100 \mathrm{mK}$ components. A $4 \mathrm{~K}$ A4K magnetic shield lines the outside wall of the $4 \mathrm{~K}$ tube, extending from the location of the second lens to the rear end of the OT. The $4 \mathrm{~K}$ and $1 \mathrm{~K}$ tubular sections were fabricated from aluminum 1100-H14 sheet to reduce thermal gradients along their lengths, which resulted in a $2 \mathrm{~K}$ temperature gradient reduction according to the simulation. The sheets were welded to $\mathrm{Al} 6061$ O-temper flanges. A final machining step is done to attain the assembly specifications. The $1 \mathrm{~K}$ components are supported from the $4 \mathrm{~K}$ tube by a

\footnotetext{
${ }^{43}$ SPIRA, http://www.spira-emi.com/.
} 
custom-made carbon fiber tube from Clearwater Composites and Van Dijk. ${ }^{44}$ A $1 \mathrm{~K}$ radiation shield is installed around the $100 \mathrm{mK}$ components. Because the shield serves the dual purpose of supporting and cooling readout components, it was fabricated out of OFHC copper. A carbon fiber truss attaches to the rear of the $1 \mathrm{~K}$ section and supports all $100 \mathrm{mK}$ components. Mechanical loading tests verified that the carbon fiber structures were able to support at least five times the expected operating loads. Simulations show the lowest resonant frequency of the carbon fiber structure is above $50 \mathrm{~Hz}$, which lies above the $30 \mathrm{~Hz}$ requirement based on our past experience with the ACT receiver.

The $100 \mathrm{mK}$ and $1 \mathrm{~K}$ cold fingers and readout support structures were all fabricated out of OFHC. The components were also gold-plated to prevent future oxidation, which would lead to reduced thermal performance. The focal plane base plates, which support the detector arrays and $100 \mathrm{mK}$ readout components, were also fabricated out of OFHC and goldplated.

\subsubsection{Detector Arrays and Readout}

The SO uses two types of dual-polarization dual-frequency TES bolometer arrays. For MF and UHF frequencies, the TES bolometers are coupled to the optics with orthomode transducers feeding monolithic feedhorn arrays, based on their well-tested performance in the Advanced ACT receiver (Duff et al. 2016; Henderson et al. 2016; Choi et al. 2020). For LF frequencies, sinuous antennas with lenslets couple the bolometers, based on the design successfully used for POLARBEAR (Suzuki et al. 2016) and the South Pole Telescope (Posada et al. 2015; Pan et al. 2018). For both architectures, each TES array is hexagon-shaped and fabricated from silicon wafers $150 \mathrm{~mm}$ in diameter. A detector array, including the light-coupling mechanism, TES bolometers, $100 \mathrm{mK}$ readout architecture, and associated magnetic shielding are packaged into a single UFM (McCarrick et al. 2021). There are three UFMs per OT. The UHF and MF OTs contain a total of 1290 pixels that couple to 5160 optically active TESes. An LF tube has 111 pixels that couple to 444 optically active TESs. Details of the UFM design are described in Li et al. (2020) and McCarrick et al (2021).

Due to space limitations, one of the most significant challenges of the OT design was the routing of the readout cabling from the three UFMs at $100 \mathrm{mK}$ to the $4 \mathrm{~K}$ components on the back of the OT magnetic shielding (Figure 10). The SO uses microwave multiplexing technology to read the detectors out (Dober et al. 2021). For the target multiplexing factor of 1000 , each OT needs 12 radio frequency (RF) coaxial cables to read out $\sim 5000$ detectors and 3 direct current (DC) ribbon cables for detector/amplifier biases and flux ramps (Mates et al. 2012).

To simplify the routing between isothermal components, hand-formable $3.58 \mathrm{~mm}$ copper coaxial cables were used. ${ }^{45}$ For connecting OTs readout components at different temperatures ( $4 \mathrm{~K}-1 \mathrm{~K}$ and $1 \mathrm{~K}-100 \mathrm{mK}$ ), low thermal conductivity semirigid cables were employed. CuproNickel (CuNi) cables with $0.86 \mathrm{~mm}$ diameter are used for $\mathrm{RF}_{\text {in }}$ to control the attenuation at each step to a colder temperature stage, as well as to limit thermal loading. For the $\mathrm{RF}_{\text {out }}$ lines, superconducting $1.19 \mathrm{~mm}$

\footnotetext{
${ }^{44}$ Van Dijk Pultrusion Products (DPP BV), https://www.dpp-pultrusion. com/en/the-company/.

45 Mini-Circuits https://www.minicircuits.com/.
}

niobium-titanium (NbTi) cables maximize signal-to-noise, yet still have good thermal isolation properties. Both types of semirigid cables are bent into loops for strain relief. DC blocks are implemented for additional electrical/thermal isolation. Attenuators on the input lines guarantee that the correct power level is delivered to the resonators, the multiplexing components in the array. Each $4 \mathrm{~K} \mathrm{RF}$ output line has an LNA mounted on the back of the magnetic shield. To reduce temperature rise due to the power generated by the LNAs ( $\sim 5 \mathrm{~mW}$ each), the LNAs are mounted on a common copper plate that has a copper strap routed down the side of each tube to the $4 \mathrm{~K}$ plate. Additional LNAs are installed at the $40 \mathrm{~K}$ stage to constitute a two-stage cold LNA system. For more details on the RF component chain, see Sathyanarayana Rao et al. (2020).

\subsubsection{Magnetic Shielding}

A layer of Cryoperm A4K manufactured by Amuneal ${ }^{46}$ covers the back of the OT to act as a $4 \mathrm{~K}$ magnetic shield, as shown in Figure 10. The A4K magnetic shield extends through the OT to the Lens 2 mounting location shown in Figure 9. A study has been conducted on the possible UFM magnetic shielding strategies for the microwave SQUID multiplexing readout system (Vavagiakis et al. 2020). As a result of this study, and in order to provide a higher shielding factor for the detector and readout components, we are also investigating plating the OT $1 \mathrm{~K}$ radiation shield with a type-I superconductor.

\subsection{Readout Interface Design}

The LATR needs to read out $>62,000$ detectors, $>120$ thermometers, 12 heaters, and two heat switches, which all require cables running from the room temperature to cryogenic temperatures. The design requires them to be routed across different temperature stages in an efficient and organized manner. There are five ports on the side of the LATR, penetrating through the $300 \mathrm{~K}$, $40 \mathrm{~K}$, and $4 \mathrm{~K}$ stages. Four of the ports are used for detector readout cables while the fifth is used for housekeeping cables (thermometers, heaters, and heat switches).

\subsubsection{Detector Readout Interface}

In the LATR, 13 OTs require 156 coaxial cables and 2400 DC wires to be routed without introducing unacceptable thermal loading. To address this, the SO developed the modularized universal readout harness (URH; Xu et al. 2020) for both the LATR and the small-aperture telescopes (Ali et al. 2020). One URH contains 48 coaxial cables penetrating three temperature stages from $300 \mathrm{~K}$ to $4 \mathrm{~K}$. In addition, each URH carries $600 \mathrm{DC}$ cryogenic wires so as to bias detectors and amplifiers, and convey flux ramps. The LATR requires four URHs to operate and read out all the detectors in 13 OTs.

Given the complexity of the cable harness and the number of cables bridging different temperature stages, uncontrolled thermal loading is a major concern. MLI blankets were tailored with minimal openings for all the cables on $40 \mathrm{~K}$ and $4 \mathrm{~K}$ plates. Since simulations on the thermal performance of the MLI sheets with many penetrations is unreliable, the URH thermal simulation only includes the thermal conductivity from the cables. The simulated thermal loading is $3 \mathrm{~W}$ (from conductive load) at the $40 \mathrm{~K}$ stage and $0.3 \mathrm{~W}$ at the $4 \mathrm{~K}$ stage.

\footnotetext{
${ }^{46}$ Amuneal Manufacturing Corp., https://www.amuneal.com/.
} 


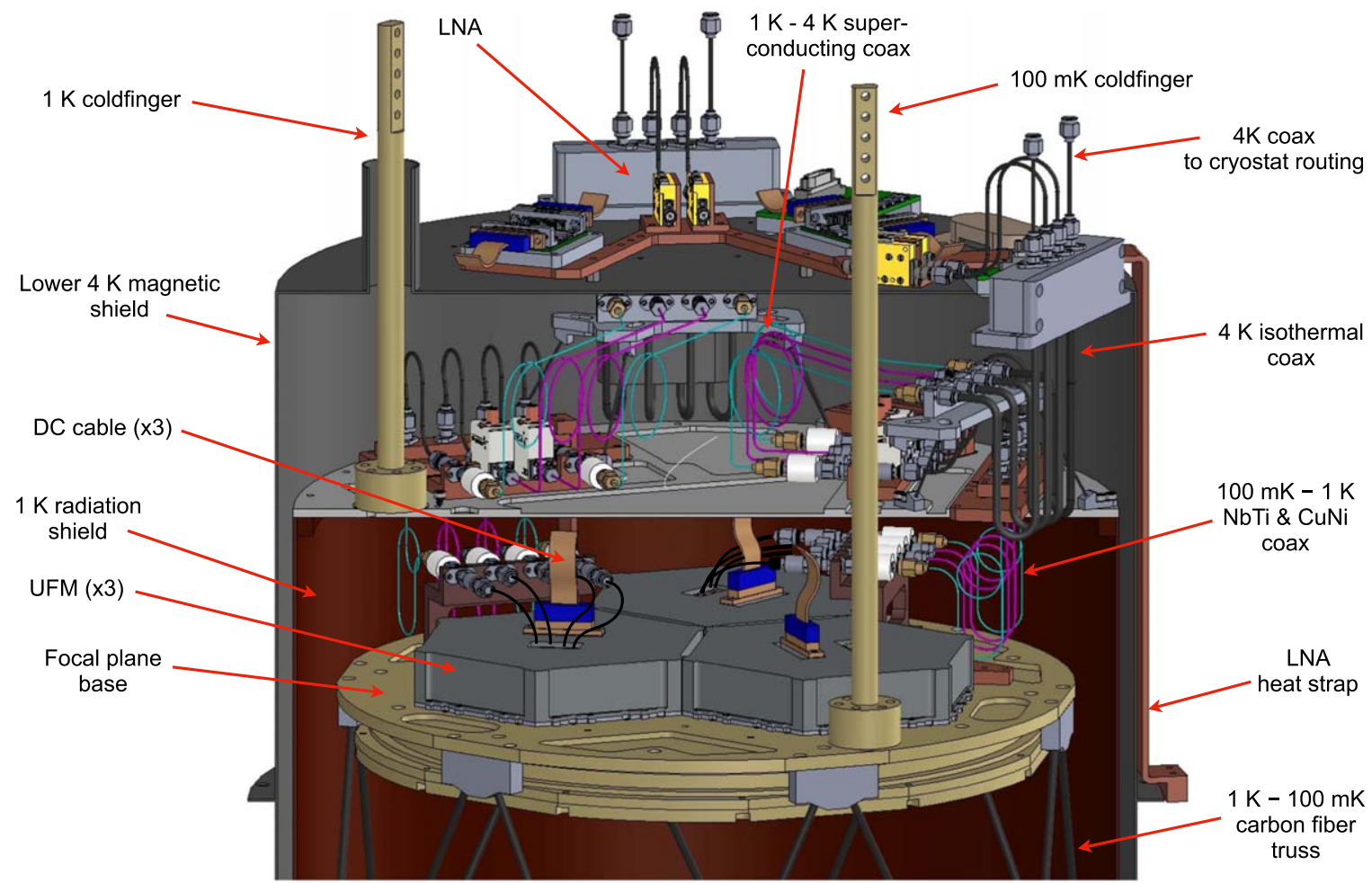

Figure 10. OT mechanical and readout components on the $4 \mathrm{~K}, 1 \mathrm{~K}$, and $100 \mathrm{mK}$ stages. This design shows the cable setup for a multiplexing factor of 1000 , where each UFM is read out by two pairs of RF lines. In addition, one DC ribbon cable per UFM provides the detector biases and flux ramps.

Measurement in another cryostat shows loading at the $40 \mathrm{~K}$ stage is $\sim 7 \mathrm{~W}$ while the loading at the $4 \mathrm{~K}$ stage is $\sim 0.15 \mathrm{~W}$. This result demonstrates the success of the MLI design at the $40 \mathrm{~K}$ stage, implying only $\sim 4 \mathrm{~W}$ of radiative load. Meanwhile, the measured $4 \mathrm{~K}$ stage thermal loading is only half of the value predicted by simulations.

All the coaxial cables and the DC wires need to leave the cryostat through the four URHs. Care was taken in the routing of the coaxial cables to satisfy space constraints while minimizing the obstruction of the $4 \mathrm{~K}$ working space by the coax. Additionally, we wanted to reduce the amount of coax that would need to be removed/installed for an OT removal/ installation, as well as reduce the complexity of those segments in order to minimize the amount of time spent removing and installing coax. Therefore, instead of directly routing individual cables for the shortest run, we ran the majority of the cables along the inside of the $4 \mathrm{~K}$ shell, minimizing the interference with the OTs. Analogous to a road system, our isothermal $4 \mathrm{~K}$ coax are designed in three parts: highways, ramps, and streets (Figure 11). The highways run along the inside of the $4 \mathrm{~K}$ shell from the URH to a set of permanently installed bulkheads, each located along the interior of the shell as close as possible to its corresponding OT. The highways are supported along their length, and constitute most of the length of the $4 \mathrm{~K}$ isothermal run (up to $1.5 \mathrm{~m}$ ). Each OT has an individual set of matching bulkheads on the back of the $4 \mathrm{~K}$ shell. The streets are permanently installed sections that run from those bulkheads to penetrations in the magnetic shielding, connecting to other cables deeper in the OT. This arrangement allows most of the complex routing to be permanently installed. Connecting the street bulkheads and the highway bulkheads are the ramp sections: short $(\sim 20 \mathrm{~cm})$ pieces of hand-formed coax. These are the only part of the isothermal $4 \mathrm{~K}$ run that are not permanently installed, and hence are the only pieces that have to be added or removed when installing or removing an OT. The DC cables run along the isothermal $4 \mathrm{~K}$ coax, and are tied down to the coaxial highways, ramps, and streets. See Figure 11 for an example of one OT's isothermal $4 \mathrm{~K}$ run.

\subsubsection{Housekeeping Readout Interface}

The LATR's temperatures are monitored with 126 thermometers, distributed across the $80 \mathrm{~K}, 40 \mathrm{~K}, 4 \mathrm{~K}, 1 \mathrm{~K}$, and $100 \mathrm{mK}$ stages. At the $80 \mathrm{~K}, 40 \mathrm{~K}$, and $4 \mathrm{~K}$ stages, we installed DT-670 silicon diodes, manufactured by Lake Shore Cryotronics. ${ }^{47}$ For the $1 \mathrm{~K}$ and $100 \mathrm{mK}$ stages, we use ruthenium oxide (ROX) sensors, ${ }^{48}$ also from Lake Shore. The diodes and ROXs were potted in custom-built copper bobbins with Stycast 2850 FT, along with heat-sinking wires and a microconnector. All thermometers are read out via a four-wire measurement, with cables manufactured by Tekdata Interconnections, Ltd. $^{49}$ The cables are routed via cryogenic breakout boards that were developed for the SO. The thermometers purchased from Lake Shore were calibrated in-house in a dedicated Bluefors DR against precalibrated reference sensors.

To carry the signal to the exterior of the LATR, we designed an assembly based on the URH design, called the housekeeping harness. Outside of the LATR, we employ Lake Shore measurement modules to do thermometry data acquisition. For $100 \mathrm{mK}$ thermometers, we utilize two Lake Shore 372 AC Resistance Bridges, each coupled to a 16-channel scanner. ${ }^{50}$ All other thermometers on higher temperature stages are read out with Lake Shore 240 Series Input Modules. All the

\footnotetext{
47 https://www.lakeshore.com/

${ }^{48}$ Model \# RX-102A-AA.

49 https://www.tekdata-interconnect.com/

50 Model 3726.
} 

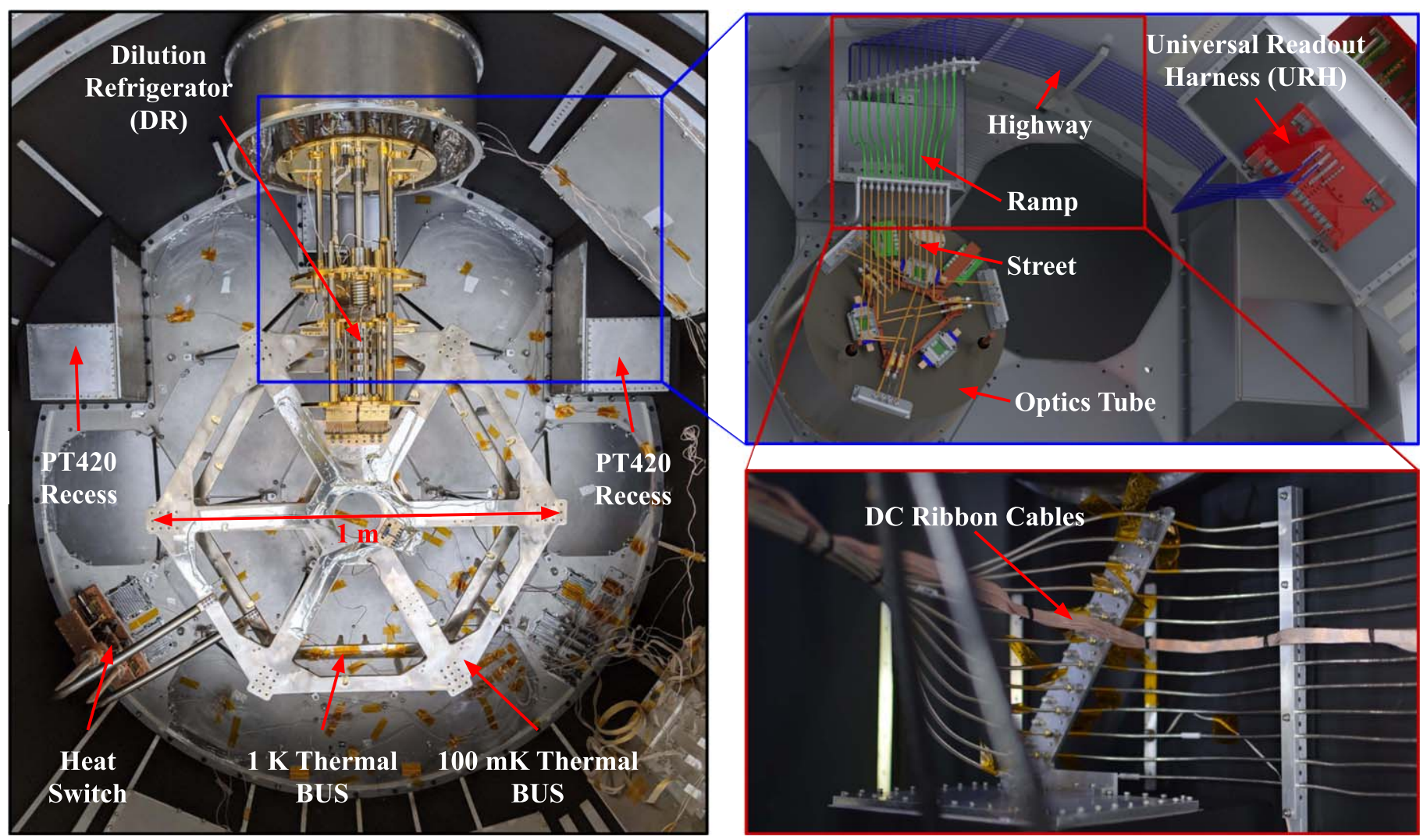

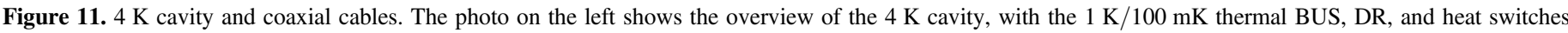

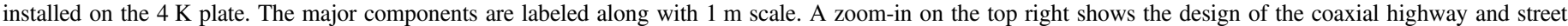

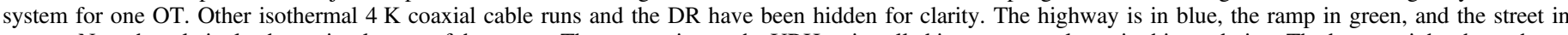

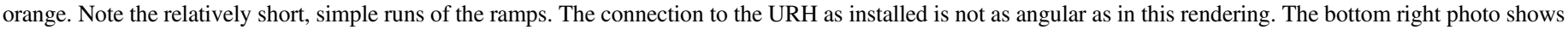
another zoom-in of the installed $4 \mathrm{~K}$ isothermal coaxial cables in the LATR.

thermometry data are read out and stored using the Observatory Control System (OCS) software developed for the SO (Koopman et al. 2020). For normal operations where the thermometers are multiplexed, they are read out in 30 second intervals. For laboratory testing where a faster readout rate is desired, a single thermometer can be read out at $>1 \mathrm{~Hz}$. The housekeeping harness is also used to monitor and control other systems, including the heat switches, low-power heaters for testing, and high-power heaters to facilitate warming up the system.

\subsection{Telescope Interface Design}

According to the LAT optics design (Dicker et al. 2018), the LATR must corotate with the telescope elevation structure as the telescope points from $0^{\circ}$ to $90^{\circ}$ in elevation (Figure 1). The corotator, as shown in Figure 12, consists of a pair of corotator rails that are bolted to the front and back flange of the LATR, and a cradle that supports and rotates the rails. The design creates a stable support structure for the LATR while allowing adjustments in the corotator cradle bases for accurate on-axis alignment. The corotation makes sure that the LATR sees the same area of the telescope mirrors at different elevations. We plan to test the corotator structure with a dummy LATR mass before deployment.

The pulse tube cooler hoses, along with all the electronic cables, converge at the cable hub on top of the cryostat (Figure 3), before going through the cable wrap. The cable wrap attaches to the cryostat, and-in a controlled manner-bends as the cryostat corotates, safely guiding the motion of the hoses and wires (Figure 1).

\section{LATR Validation and Tests}

During the assembly of the LATR, we performed several rounds of metrology to guarantee the stringent alignment requirements were achieved under different loads. We also measured the resonant frequency of the $1 \mathrm{~K}$ and $100 \mathrm{mK}$ thermal BUS stages with accelerometers at room temperature.

The cryogenic properties of the LATR were systematically tested in multiple configurations, including dark tests, optical tests, and tests with OTs installed. We tilted the entire cryostat to test its performance at different orientations as well as to determine the thermal time constant of the system.

After the cryostat and first OT were successfully integrated and tested for mechanical and cryogenic performance, the RF chain for reading out the detectors was connected (and looped back at the $100 \mathrm{mK}$ stage). The RF performance will be covered in a future publication.

\subsection{Mechanical Validation}

Comprehensive simulations were performed to set the required tolerance for the alignment of the LATR optical components (Dicker et al. 2018). The location of the LATR is referenced to the telescope-receiver interface: the corotator. Utilizing a reference plane that is external to the receiver shell ensures that the optical components within the LATR will be aligned with the primary and secondary mirrors in the LAT. The positions of optical components within the LATRincluding the lenses, filters, and detectors-must adhere to tight 


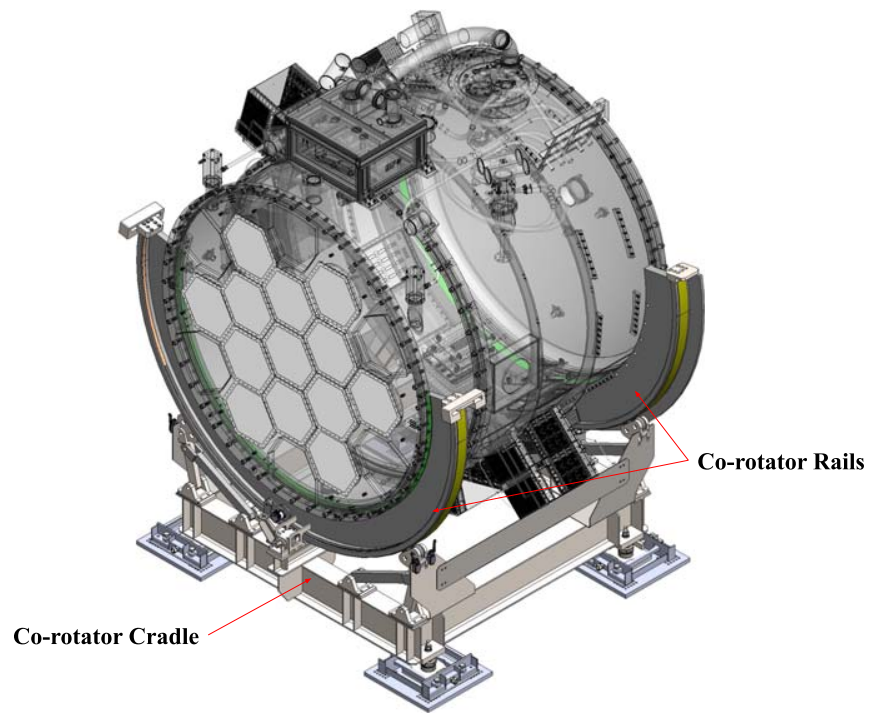

Figure 12. The LATR corotator. The corotator rails are bolted to the front and back flange of the cryostat. The four feet of the corotator cradle allow for fine adjustment when aligning the cryostat with the secondary mirror. In this rendering, the cryostat is transparent to showcase the corotator parts.

tolerance requirements, both statically and dynamically (i.e., during corotation).

Optimization of Strehl ratios using optical simulations (Dicker et al. 2018) lead to tight physical constraints being placed on the locations of our OTs and cryogenic lenses. The position of the OTs must be maintained within $\pm 3 \mathrm{~mm}$ along the LATR's optical axis and $\pm 3 \mathrm{~mm}$ perpendicular to the LATR's optical axis. In addition, the OTs must not exceed \pm 0.8 in tilt with respect to each other. These constraints need to be met when the cryostat is initially deployed with a small number of OTs and also when it is fully loaded with 13 OTs. The requirement must also be maintained when the LATR is rotated.

To ensure these tight tolerances were met, we utilized the FARO Vantage Laser Tracker ${ }^{51}$ to measure the locations of all relevant surfaces both internal to, and external to, the cryostat at room temperature. The FARO Vantage Laser Tracker has an accuracy of $16 \mu \mathrm{m}$ and a single-point repeatability of $8 \mu \mathrm{m}$ at $1.6 \mathrm{~m}$.

The Vantage Laser Tracker was used to verify the overall dimensions of the cryostat, the 3D locations of each of the four temperature stages, and the precise locations each OT will inhabit when installed. Due to the nature of the cryostat and the support provided by the $4 \mathrm{~K}$ stage, it was important for us to measure the location of the $4 \mathrm{~K}$ stage both with and without the load of 13 OTs. Upon measuring the various temperature stages with no load on the $4 \mathrm{~K}$ stage, we were able to conclude that the positions of our OTs would be well within our required tolerances. A detailed list of the measured position of the $4 \mathrm{~K}$ plate without OTs can be found in Table 4.

We were also able to perform measurements of the $4 \mathrm{~K}$ stage with 13 OT mass dummies installed. These mass dummies accurately reproduced both the total mass and the location of the center of mass for each OT. Once all 13 mass dummies were installed, the metrology process was repeated. Even with all 13 mass dummies added, the $4 \mathrm{~K}$ plate remained within the required positional tolerances. A detailed list of the measurements recorded for the loaded $4 \mathrm{~K}$ plate can be found in Table 4 . As can be seen from the measurements, the difference between the loaded and the

\footnotetext{
${ }^{51}$ FARO, https://www.faro.com/.
}

Table 4

LATR 4 K Plate Metrology Results

\begin{tabular}{lccc}
\hline \hline Axis & Tolerance & $\begin{array}{c}\text { Deviation from } \\
\text { Origin (No Load) }\end{array}$ & $\begin{array}{c}\text { Deviation from Origin } \\
\text { (13 OT Load) }\end{array}$ \\
\hline$X$-axis & $3 \mathrm{~mm}$ & $0.49 \pm 0.03 \mathrm{~mm}$ & $0.31 \pm 0.02 \mathrm{~mm}$ \\
$Y$-axis & $3 \mathrm{~mm}$ & $2.04 \pm 0.03 \mathrm{~mm}$ & $2.24 \pm 0.02 \mathrm{~mm}$ \\
$Z$-axis & $3 \mathrm{~mm}$ & $2.03 \pm 0.03 \mathrm{~mm}$ & $2.43 \pm 0.02 \mathrm{~mm}$ \\
\hline
\end{tabular}

Note. This table shows the position of the $4 \mathrm{~K}$ plate under two configurations: no load and with load. No load measurements refer to those performed with no OT mass dummies installed. Loaded measurements refer to those performed with 13 OT mass dummies installed. Under both conditions, the plate is within the required tolerances provided by Dicker et al. (2018). The nominal origin, and thus center of the $4 \mathrm{~K}$ plate, is assumed to be $(0,0,0)$ in a $3 \mathrm{D}$ coordinate system. Looking at the cryostat from a viewpoint in front of it, $Z$-axis is the optical axis, $Y$-axis is pointing toward the ground, and $X$-axis is pointing toward the right. All deviation values are absolute values of the measured deviations of the center of the $4 \mathrm{~K}$ plate from the origin.

Table 5

LATR Front Plate Metrology Results-Under Vacuum

\begin{tabular}{lcc}
\hline \hline Axis & $\begin{array}{c}\text { Deviation from Origin } \\
\text { (Simulated) }\end{array}$ & $\begin{array}{c}\text { Deviation from Origin } \\
\text { (Measured) }\end{array}$ \\
\hline$X$-axis & $0 \mathrm{~mm}$ & $0.22 \pm 0.05 \mathrm{~mm}$ \\
$Y$-axis & $0 \mathrm{~mm}$ & $0.19 \pm 0.05 \mathrm{~mm}$ \\
$Z$-axis & $18.1 \mathrm{~mm}$ & $17.2 \pm 0.03 \mathrm{~mm}$ \\
\hline
\end{tabular}

Note. This table highlights the expected and measured position of the LATR's front plate under vacuum. The expected position of the front plate under vacuum is provided by the simulation performed in (Orlowski-Scherer et al. 2018) and shown in Table 5. The nominal origin, and thus center of the front plate, is assumed to be $(0,0,0)$ in a $3 \mathrm{D}$ coordinate system. All deviation values are absolute values of the measured deviations of the center of the front plate from the origin.

unloaded measurements is less than $0.5 \mathrm{~mm}$, which is within specifications. The overall offset in the $Y$ and $Z$ axes is likely due to buildup of manufacturing tolerances. The $Z$-axis offset can be removed by refocusing. The $Y$-axis offset could be removed by shifting the $4 \mathrm{~K}$ cold plate, if necessary.

The Vantage Laser Tracker system was also used to measure the LATR's front plate while under vacuum to ensure that the deformation of the plate was within the expected regime. Simulations of the deformation of the front plate can be found in Table 5 along with the measurement results. From these results, we can see that the measured deformation along the Zaxis, or bowing, was within $1 \mathrm{~mm}$ of the simulated result (slightly smaller than expected).

The optical simulations performed also provided constraints on our lens positions within the OT themselves. All three lenses within the OTs must maintain a position of $\pm 2 \mathrm{~mm}$ along the LATR's optical axis and $\pm 2 \mathrm{~mm}$ perpendicular to the LATR's optical axis. The tilt of each lens must not exceed \pm 0.4 with respect to the axis perpendicular to the optical path. The focal plane array within the OT must maintain a position of $\pm 2.5 \mathrm{~mm}$ along the LATR's optical axis. Further constraints placed on the beam guiding $80 \mathrm{~K}$ alumina wedges and the surface accuracy of the lenses can be found in Dicker et al. (2018).

Due to the size and intricate design of the OTs, we utilized the FARO Edge ScanArm to obtain precise cold optical component and detector array locations. The lens and OT metrology was performed on (warm) tubes before they were installed in the LATR, to allow for easier access to hard-to-reach locations within 
Table 6

An Example of LATR Optics Tube Metrology Results

\begin{tabular}{lcc}
\hline \hline $\begin{array}{l}\text { Optical } \\
\text { Element }\end{array}$ & $\begin{array}{c}\text { Z-axis } \\
\text { Tolerance }\end{array}$ & $\begin{array}{c}\text { Deviation } \\
\text { from Design }\end{array}$ \\
\hline Lens 1 & $2.0 \mathrm{~mm}$ & $0.47 \pm 0.02 \mathrm{~mm}$ \\
Lens 2 & $2.0 \mathrm{~mm}$ & $0.19 \pm 0.02 \mathrm{~mm}$ \\
Lens 3 & $2.0 \mathrm{~mm}$ & $0.50 \pm 0.02 \mathrm{~mm}$ \\
Lyot Stop & $2.0 \mathrm{~mm}$ & $0.22 \pm 0.02 \mathrm{~mm}$ \\
Detector Arrays & $2.5 \mathrm{~mm}$ & $0.53 \pm 0.02 \mathrm{~mm}$ \\
\hline
\end{tabular}

Note. This table reports the positions of the optical elements within a LATR OT. The $z$-axis travels along the central axis of the cylindrical OT. The listed tolerances are provided by Dicker et al. (2018). All deviation values are absolute values of the measured deviations of central plane of each element, perpendicular to the central axis, with respect to the designed central plane for each element.

the tubes. The FARO Edge ScanArm has an accuracy of $34 \mu \mathrm{m}$ and a repeatability of $24 \mu \mathrm{m}$ at $1.8 \mathrm{~m}$. Each individual mechanical component of every OT is measured and recorded. Partial and full subassemblies of the OT are also measured, thus allowing us to understand the position of, parallelism between, distance between, and coaxiality of all components within each OT. Tight constraints on the locations of the focal plane array and lenses created a need for extensive documentation of all dimensions of OT components. Measurements of the positions of the optical elements and their deviations from designed locations can be found in Table 6. From these measurements, we have deduced that all of the lenses, the Lyot stop, and the focal plane in the first optical tube are located within the required tolerances.

Since the corotator on the LAT is not yet available, we are unable to measure the LATR at a variety of different clocking orientations. However, the differences between the unloaded and loaded displacements provided in Table 4 suggest that optical alignment will be well within specification.

Beyond the work on the LATR, the FARO Vantage Laser Tracker will also be used within the LAT to ensure the proper positioning of the mirror panels for the telescope's primary and secondary mirrors.

\subsection{Cryogenic Validation}

In order to know the total thermal loading on each stage precisely, we calibrated all four pulse tube coolers individually in a smaller and well-understood cryostat, measuring their specific cooling power at different temperatures. The information enabled us to accurately deduce the thermal loading on each stage by closely monitoring the pulse tube cold head temperatures.

The cryogenic validation of the LATR proceeded in incremental steps, starting with the simplest configuration and gradually adding components. We first conducted $4 \mathrm{~K}$ dark tests, with two PT420s and two PT90s. Following completion of this test, we installed the Bluefors DR with the associated thermal straps that coupled the DR to the cryostat $4 \mathrm{~K}$ shell and the $1 \mathrm{~K} / 100 \mathrm{mK}$ thermal BUS. From the base temperatures in the dark tests, we backed out the loading on each stage using the cryogenic cooler load curves we calibrated during the preparation. These two rounds of dark tests provide a baseline loading without external radiation.

After the dark tests, we installed $300 \mathrm{~K}$ windows made out of $1 / 4$ inch ultra-high-molecular-weight polyethylene sheets. Behind each window, a double-sided infrared blocker (DSIR) at $300 \mathrm{~K}$ was installed on the back of the front plate to reflect out-of-band radiative power. Another DSIR filter was installed on the $80 \mathrm{~K}$ stage, followed by an AR-coated alumina filter (Nadolski et al. 2018) also at $80 \mathrm{~K}$. The alumina filter absorbs the residual infrared radiation and conducts the heat away efficiently. After the alumina filter, another DSIR, at $40 \mathrm{~K}$, reflects the residual infrared radiation before it enters the $40 \mathrm{~K}$ cavity. The $40 \mathrm{~K}$ cavity then houses the front part of the 13 OTs, including the filters and lenses at $4 \mathrm{~K}$ and below (Figure 4). Prior to installing OTs, metal blanks with MLI were installed on the $4 \mathrm{~K}$ plate to block the radiation from the $40 \mathrm{~K}$ cavity.

We cryogenically tested the LATR with two windows installed ("2-window" configuration) and subsequently with three windows installed ("3-window" configuration). The "3-window" configuration also had one OT and one URH installed. Given the overall progress of the project, initially we only fabricated three sets of the filters to give us enough fidelity to extrapolate the performance with all 13 windows installed.

Between the $80 \mathrm{~K}, 40 \mathrm{~K}$, and $4 \mathrm{~K}$ stages, the $80 \mathrm{~K}$ is the most sensitive to radiation loading because of the absorptive $80 \mathrm{~K}$ alumina filter. Measurements of the $80 \mathrm{~K}$ stage thermal loading under different configurations are summarized in Table 7 . The baseline loading from the dark test is $\sim 22 \mathrm{~W}$, with each window adding another $\sim 7 \mathrm{~W}$ radiation loading on the $80 \mathrm{~K}$ stage. If we project the results to 13 windows, the anticipated loading will be $\sim 113 \mathrm{~W}$. Given the load curve of the PT90s and thermal strap conductance, we calculate that the $80 \mathrm{~K}$ stage will stay at around $65 \mathrm{~K}$, safely below the designed $80 \mathrm{~K}$ requirement. Because the $80 \mathrm{~K}$ filter plate is made of thermally conductive aluminum 1100 series, the temperature gradient across the $2.1 \mathrm{~m}$ diameter plate measured $\sim 3 \mathrm{~K}$ with three windows installed. Receiving roughly three times the loading with 13 OTs, the gradient may increase to $\sim 9 \mathrm{~K}$, still less than the designed requirement at $\sim 10 \mathrm{~K}$ (Figure 7 ).

While the observed loading in Table 7 meets the LATR's requirements, it is higher than the models predicted. In the dark configuration, this is likely due to imperfections in the MLI. Considering the size of the $2.1 \mathrm{~m}$ diameter $80 \mathrm{~K}$ filter plate and the complicated geometry of the G-10 tabs, it is unsurprising to observe extra loading due to imperfect shielding of all the surfaces. In the window tests, we measured $\sim 7 \mathrm{~W}$ per filter set instead of the predicted $\sim 3 \mathrm{~W}$. We are still investigating the mismatch, but it is likely a result of higher-than-expected infrared transmission of the DSIR filters. Another factor that should be considered is that all the measurements were performed when both of the pulse tube coolers were operating at $<40 \mathrm{~K}$, much lower than their nominal $80 \mathrm{~K}$ operation temperature. This is near the lower limit of the temperature the pulse tube coolers can reach, where only a sparsely sampled calibration curve is available. This adds $\mathcal{O}(1 \mathrm{~W})$-level uncertainties in the measurements.

During the dark test, the $40 \mathrm{~K}$ filter plate stayed at $44-47 \mathrm{~K}$ (measured at six locations on the plate) with an estimated loading of $33 \pm 1 \mathrm{~W}$; the $4 \mathrm{~K}$ plate stayed at $3.5-5.2 \mathrm{~K}$ (measured at five locations on the plate) with an estimated loading of $0.8 \pm 0.1 \mathrm{~W}$ (Table 8). Estimating the thermal loading, especially on the $4 \mathrm{~K}$ stage, is challenging at these very low power levels where the calibration of the pulse tubes is less certain and thus results in a $\sim 1 \mathrm{~W}$ loading uncertainty on the $40 \mathrm{~K}$ stage and $\sim 0.1 \mathrm{~W}$ on the $4 \mathrm{~K}$ stage. To achieve a more accurate measurement, we installed seven heaters (evenly distributed) on the $4 \mathrm{~K}$ plate to add additional power and monitored the temperatures on the stage. From the data, we calculated the thermal conductance of the thermal straps connecting the pulse tube $4 \mathrm{~K}$ cold heads to the cryostat $4 \mathrm{~K}$ plate and then backed out what the thermal loading 
Table 7

80 K Stage Thermal Performance

\begin{tabular}{lccc}
\hline \hline $\begin{array}{l}\text { Test } \\
\text { Configuration }\end{array}$ & $\begin{array}{c}\text { Filter-plate } \\
\text { Temperature }\end{array}$ & $\begin{array}{c}\text { Measured } \\
\text { Loading }\end{array}$ & $\begin{array}{c}\text { Predicted } \\
\text { Loading }\end{array}$ \\
\hline Dark & $37-39 \mathrm{~K}$ & $22 \mathrm{~W}$ & $10.1 \mathrm{~W}$ \\
2-window & $40-43 \mathrm{~K}$ & $35 \mathrm{~W}$ & $17.9 \mathrm{~W}$ \\
3-window & $44-47 \mathrm{~K}$ & $42 \mathrm{~W}$ & $21.8 \mathrm{~W}$ \\
7-window* & $51 \mathrm{~K}^{*}$ & $71 \mathrm{~W}^{*}$ & $37.3 \mathrm{~W}$ \\
13-window $^{*}$ & $65 \mathrm{~K}^{*}$ & $113 \mathrm{~W}^{*}$ & $60.6 \mathrm{~W}$ \\
\hline
\end{tabular}

Note. Base temperature and thermal loading for the $80 \mathrm{~K}$ stage under different configurations. The temperature range is measured from six thermometers evenly distributed on the $80 \mathrm{~K}$ filter plate. The starred values are extrapolated from measured results. The predicted load is derived from our thermal model in Table 3. Cooling power on the $80 \mathrm{~K}$ stage was designed with a significant margin. The difference between the predicted loading and the measured loading is explained in the text.

would be without additional loading from the heaters. This independent measurement gives results consistent with those from the PT420 load curves.

After the DR was installed (starting from the "2-window" configuration in Table 8), estimating thermal loading on the $40 \mathrm{~K}$ and $4 \mathrm{~K}$ stage became more difficult because the PT420 on the DR also contributed to the cooling of the $40 \mathrm{~K}$ and $4 \mathrm{~K}$ stages. We did not calibrate the PT420 in the DR since it is deeply integrated in the DR system. Furthermore, the conductivity of the thermal links between the DR $40 \mathrm{~K} / 4 \mathrm{~K}$ stages and the main cryostat $40 \mathrm{~K} / 4 \mathrm{~K}$ stages are hard to quantify. The DR and the cryostat $40 \mathrm{~K}$ stages are connected by aluminum-coated mylar tape that is intended only for sealing the gaps between them to stop the light leak. The DR and the cryostat $4 \mathrm{~K}$ stages are connected by $5 \times 10$-stacked highpurity $(99.999 \%)$ aluminum tabs. The aluminum tabs bridge the $\sim 6 \mathrm{~mm}$ gap, and have dimensions of $0.5 \times 50 \times 75 \mathrm{~mm}$. We measured the $40 \mathrm{~K}$ and $4 \mathrm{~K}$ stage temperatures of the DR PT420 and compared to the values previously measured in its designated cryostat as a no-extra-load reference. The DR PT420 $4 \mathrm{~K}$ temperature did not change in the "2-window" configuration and rose by $\sim 0.2 \mathrm{~K}$ in the "3-window" configuration, mainly because of the addition of the OT and the URH. Using the average of the calibrated load curves from the two PT420s, we estimated the additional cooling power due to the DR PT420, and thus the total loading from the observed temperature change. The thermal loading measurements are reported in Table 8.

Interestingly, the DR PT420 $40 \mathrm{~K}$ temperature stage decreased after installation in the LATR. This means that the main cryostat $40 \mathrm{~K}$ stage cools to a lower temperature than the DR $40 \mathrm{~K}$ stage, such that it is "lending" cooling power to the DR $40 \mathrm{~K}$ stage when thermally connected. Therefore, we report the $40 \mathrm{~K}$ thermal loading from the two calibrated PT420s as upper limits in Table 8.

The temperature gradient across the $2.06-\mathrm{m}$ diameter $40 \mathrm{~K}$ filter plate is $\leqslant 4 \mathrm{~K}$ with three windows. From the "dark" to the "3window" configuration, we did not measure significant changes on the $40 \mathrm{~K}$ filter plate in terms of thermal loading and thermal gradient. Based on that observation, the simulation is validatedshowing the $40 \mathrm{~K}$ filter plate will not change significantly with a fully equipped LATR, largely because only reflective filters are installed on this stage. Additionally, the $40 \mathrm{~K}$ stage of the URH measured $\sim 50 \mathrm{~K}, 4 \mathrm{~K}$ higher than the $40 \mathrm{~K}$ filter-plate average. It is the warmest part of the $40 \mathrm{~K}$ stage since it is both far away from the pulse tube cold heads (Figure 11) and the source of significant thermal loading $(\sim 7 \mathrm{~W}$ for $4 \mathrm{URHs}$ for the fully loaded
Table 8

40 K/4 K Stage Thermal Performance

\begin{tabular}{lcll}
\hline \hline $\begin{array}{l}\text { Test } \\
\text { Configuration }\end{array}$ & $\begin{array}{c}\text { Plate } \\
\text { Temperature }\end{array}$ & Power & $\begin{array}{r}\text { Predicted } \\
\text { Loading }\end{array}$ \\
\hline \multicolumn{4}{c}{40 K Filter Plate } \\
\hline Dark & $44-47 \mathrm{~K}$ & $33_{-1}^{+1} \mathrm{~W}$ & $35.3 \mathrm{~W}$ \\
2-window & $44-47 \mathrm{~K}$ & $<33 \mathrm{~W}$ & $35.3 \mathrm{~W}$ \\
3-window + 1-OT & $44-48 \mathrm{~K}$ & $<34 \mathrm{~W}$ & $38.8 \mathrm{~W}$ \\
\hline \multicolumn{5}{c}{$4 \mathrm{~K} \mathrm{Plate}$} & & \\
\hline Dark & $3.5-5.2 \mathrm{~K}$ & $0.8_{-0.1}^{+0.1} \mathrm{~W}$ & $0.42 \mathrm{~W}$ \\
2-window & $3.6-5.0 \mathrm{~K}$ & $0.8_{-0.1}^{+0.2} \mathrm{~W}$ & $0.47 \mathrm{~W}$ \\
3-window + 1-OT & $3.8-5.2 \mathrm{~K}$ & $1.3_{-0.2}^{+0.2} \mathrm{~W}$ & $0.75 \mathrm{~W}$ \\
\hline
\end{tabular}

Note. Base temperature and loading for the $40 \mathrm{~K}$ filter plate and the $4 \mathrm{~K}$ plate under different configurations, based on measurements at multiple locations. In the "dark" configuration, the two calibrated PT420s cooled the $40 \mathrm{~K}$ and $4 \mathrm{~K}$ stages; starting from the "2-window" configuration, the PT420 on the DR was thermally connected to the main cryostat in $40 \mathrm{~K} / 4 \mathrm{~K}$ stages. We estimated contribution from the DR PT420 by comparing its temperatures in different configurations to the ones measured in its standalone cryostat. We used the average of the two calibrated PT420s and estimated the power given the temperature change on the $4 \mathrm{~K}$ stage. However, the DR PT420 $40 \mathrm{~K}$ temperature decreased after it was installed in the main cryostat (being cooled by the other two PT420s), thus we report the number from the other two PT420s as upper limits. In the "3-window" configuration, one OT and one URH were also installed in addition to three windows.

configuration). The measured temperatures and the small thermal gradient proved that the pulse tube cooling power is efficiently distributed around the $2 \mathrm{~m}$ long $40 \mathrm{~K}$ stage to maintain the entire stage below $50 \mathrm{~K}$.

The thermal gradient across the $4 \mathrm{~K}$ plate is around $1.7 \mathrm{~K}$ in the "dark" configuration, and reduced to $1.4 \mathrm{~K}$ after the addition of the DR. The difference between the measured and predicted loading may come from the decreased fidelity of the simulation at the low-power limit. The addition of the windows did not significantly change the loading on the $4 \mathrm{~K}$ which is consistent with the simulation. In the "3-window" configuration, the temperature on the $4 \mathrm{~K}$ shell is $\sim 5 \mathrm{~K}$, including the $4 \mathrm{~K}$ part of the URH. This measurement validates that the entire $4 \mathrm{~K}$ stage is efficiently cooled with 3 windows, one OT, and one URH installed, and we see no evidence that the full complement of OTs will introduce unacceptable loading.

Enclosed within the $4 \mathrm{~K}$ cavity, the $100 \mathrm{mK}$ thermal BUS cooled to $<50 \mathrm{mK}$ with the $1 \mathrm{~K}$ thermal BUS maintained at $\sim 1 \mathrm{~K}$. Since we do not have 13 OTs during these tests, heaters were installed on both the $100 \mathrm{mK}$ and $1 \mathrm{~K}$ thermal BUS to simulate thermal loads. With these two heaters, we were able to map out the load curve on the two stages which later informed our understanding of loading from the installed OTs. We also applied the anticipated loading from 13 OTs on the two stages, and the $100 \mathrm{mK}$ thermal BUS stayed below $100 \mathrm{mK}$ with $\lesssim 10 \mathrm{mK}$ thermal gradient across its diameter, meeting the requirement.

The cooldown time for each temperature stage changes with the configuration of the LATR. Intuitively, the more thermal mass we add and the more thermal loading we introduce, the longer the cooldown process will take. Currently, the most comprehensive test we have performed is the configuration with three windows (and the corresponding filters at $300 \mathrm{~K}$, $80 \mathrm{~K}$, and $40 \mathrm{~K}$ ) and one OT. 

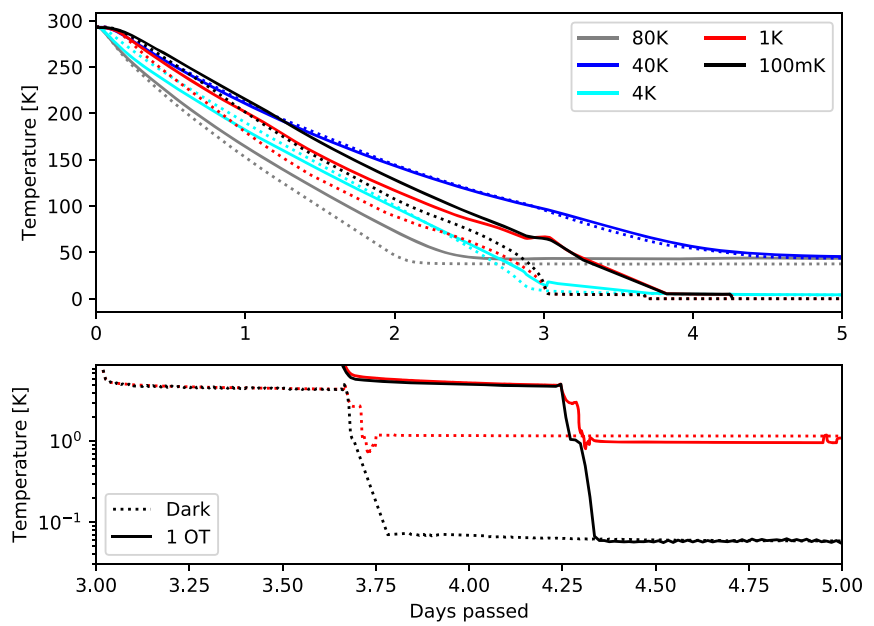

Figure 13. Cooldown curves at each temperature stage from a test run including one OT and three optical windows (and filters). Although multiple thermometers are installed on each temperature stage, we show only one representative thermometer per stage. Temperatures measured during the dark cooldown are represented by dotted lines, and during the three window cooldown as solid lines. In both configurations, all the stages cooled to base temperatures within five days. The bottom panel is a zoomed-in logarithmic plot of the $1 \mathrm{~K}$ and $100 \mathrm{mK}$ stages. These two stages reached base temperatures within hours of the dilution refrigerator being turned on.

The first OT was installed at the center of the cryostat behind one of the three window+filter assemblies. During the cooldown, temperatures of different stages as a function of time were recorded. Figure 13 shows the cooldown curve from representative thermometers on all temperature stages.

To evacuate the LATR, we initially pump with two Edwards $^{52}$ XDS35i roughing pumps through two KF40 flanges, one at the front of the cryostat and one at the back. This process takes about 1.5-2.5 days, depending on the humidity. We then reach high vacuum using an Edwards nEXT400 turbo pump bolted directly to an ISO 100 flange at the back of the LATR. Reaching high vacuum from rough vacuum takes about $4 \mathrm{hr}$, so the whole process takes 2-3 days.

After the cryostat reached high vacuum, we turned on all five pulse tube coolers at the same time. In this configuration, the $80 \mathrm{~K}$ stage was able to reach its base temperature within three days and the $4 \mathrm{~K}$ stage was able to reach its base temperature within four days, as shown in Table 9. After the $4 \mathrm{~K}$ stage reached its base temperature, the DR began operating, cooling the $1 \mathrm{~K}$ and $100 \mathrm{mK}$ stages to base temperatures within several hours (Figure 14). The $40 \mathrm{~K}$ stage, in particular the $40 \mathrm{~K}$ filter plate, reached base temperature in five days because it is the farthest from the PT420 cold heads (Figure 4). The observed cooldown times of the various stages with a $4 \mathrm{~K}$ cold mass were expected and reflected the simulated performance. Also shown in Table 9 is the base temperature for each stage-measured either on the plate or on the BUS - along with the corresponding thermal gradient.

Thermal loading on the $100 \mathrm{mK}$ stage was increased by $<4 \mu \mathrm{W}$ after installing one OT in the center position. With the amount of added power being so small compared to the rest of the $100 \mathrm{mK}$ structure, it is hard to obtain a more accurate loading value from a single OT. With the addition of active readout components and optical loading during operation, the total expected loading from one OT is $\lesssim 5 \mu \mathrm{W}$ (Harrington et al. 2020; Xu et al. 2020). Extrapolating from this number, the

\footnotetext{
${ }^{52}$ Edwards Vacuum, https://www.edwardsvacuum.com/.
}

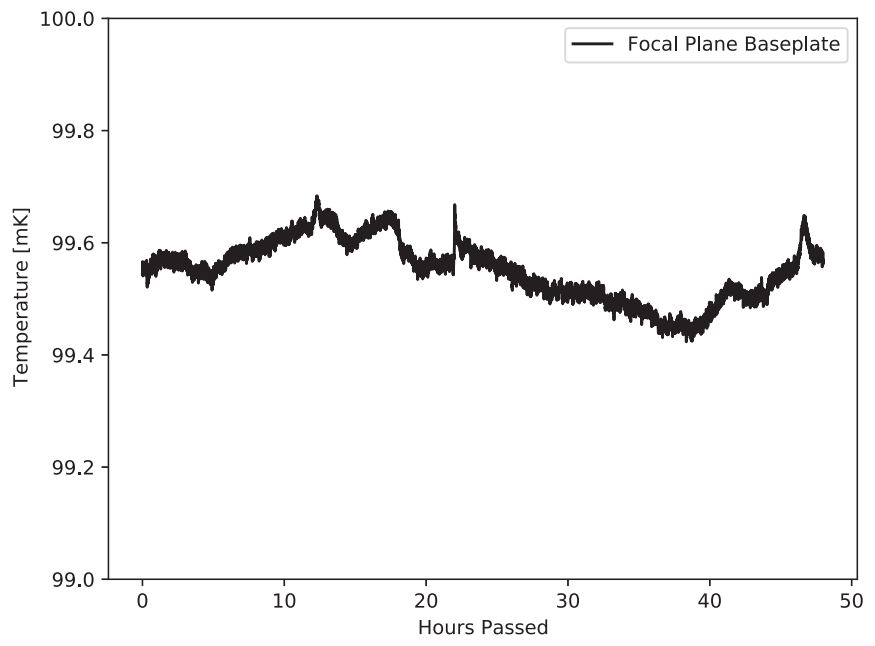

Figure 14. The temperature of an OT $100 \mathrm{mK}$ stage over $48 \mathrm{hr}$. On this timescale, the OT focal plane stage temperature varies by $\lesssim 0.3 \mathrm{mK}$. At this level, bath temperature fluctuations will not meaningfully detract from overall detector stability.

Table 9

LATR Cooldown Time

\begin{tabular}{lccc}
\hline \hline $\begin{array}{l}\text { Temperature } \\
\text { Stage }\end{array}$ & $\begin{array}{c}\text { Cooldown } \\
\text { Time }\end{array}$ & $\begin{array}{c}\text { Plate/BUS } \\
\text { Temperature }\end{array}$ & $\begin{array}{c}\text { Thermal } \\
\text { Gradient }\end{array}$ \\
\hline $80 \mathrm{~K}$ & 3 days & $43 \mathrm{~K}$ & $\pm 1 \mathrm{~K}$ \\
$40 \mathrm{~K}$ & 5 days & $47 \mathrm{~K}$ & $\pm 3 \mathrm{~K}$ \\
$4 \mathrm{~K}$ & 4 days & $4.5 \mathrm{~K}$ & $\pm 0.7 \mathrm{~K}$ \\
$1 \mathrm{~K}$ & 5 days & $1.2 \mathrm{~K}$ & $\pm 0.07 \mathrm{~K}$ \\
$100 \mathrm{mK}$ & 5 days & $55 \mathrm{mK}$ & $\pm 10 \mathrm{mK}$ \\
\hline
\end{tabular}

Note. Cooldown time measured in the 3-window configuration with one OT installed. This is not to be confused with the simulated cooldown with 13 OTs, which will take significantly longer due to added cold mass.

estimated extra loading from 13 OTs will be $\sim 70 \mu \mathrm{W}$. Since we did not have all 13 OTs available, we installed a heater on the $100 \mathrm{mK}$ thermal BUS stage and dissipated $65 \mu \mathrm{W}$ to simulate anticipated thermal loading from the other 12 OTs that are not installed. Although concentrating 13 OTs loading to one location will not reflect the actual loading distribution, the purpose of this test was to ensure the proper performance of the $100 \mathrm{mK}$ thermal BUS. The $100 \mathrm{mK}$ thermal BUS was designed to effectively distribute the cooling power to 13 OTs. When the $70 \mu \mathrm{W}$ total loading was applied, the thermal BUS temperature rose to $78 \mathrm{mK}$ at the coldest point (closest to the cold strap) and $87 \mathrm{mK}$ at the hottest point (furthest from the cold strap). In order to ensure that these temperatures were acceptable, we also needed to inspect the thermal gradient between the thermal BUS and OT $100 \mathrm{mK}$ stage. The target temperature for a focal plane baseplate in the fully loaded 13 OT configuration is $<100 \mathrm{mK}$. We observed that the gradient between thermal BUS and OT $100 \mathrm{mK}$ stage was $\sim 5 \mathrm{mK}$. This means that when we extrapolate the loading to 13 OTs, the warmest OT focal plane stage will be at most $92 \mathrm{mK}$, accounting for the uncertainty in our measurement of the $100 \mathrm{mK}$ loading from one OT. This implies that the LATR will meet the required thermal specifications.

After ensuring that the LATR would be able to thermally support 13 OTs, we moved onto testing the thermal performance of the OTs themselves. In order to maintain the required thermal 


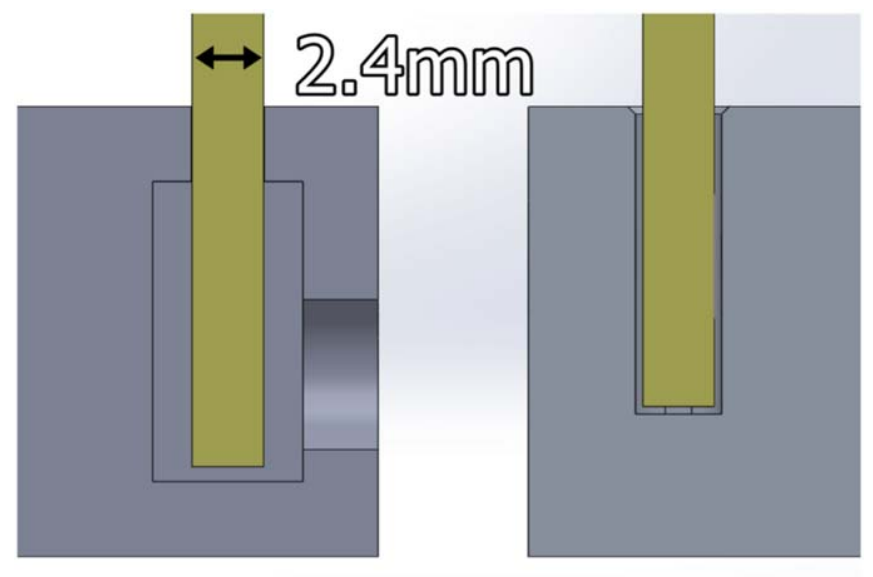

Figure 15. Recommended (left) and installed (right) G-10 tab glue joint designs, shown as a cross section of the tab foot. The scale is slightly different between the two, but the G-10 tab (yellow) is the same size. The aluminum foot is in gray. The cavity to the left in the recommended design is a glue injection passage. Note the overhang by the aluminum foot of the glue join in the recommended design, and the much tighter fit of the G-10 tab.

environment for the detector arrays, we needed to ensure the OT $100 \mathrm{mK}$ stage had both long-term temperature stability and a negligible thermal gradient across that stage. Long-term temperature stability is important to detector performance, as bath temperature drifts can impact detector data quality. Consistent thermal bath temperature between adjacent detector arrays is also important to ensure the best quality of detector data. In Figure 14, we show how the temperature of one OT $100 \mathrm{mK}$ stage changes over the course of $48 \mathrm{hr}$. Similar to the thermal tests described thus far, the OT was dark-with the $4 \mathrm{~K}$ and $1 \mathrm{~K}$ stages blanked off. Constant power was applied to a heater near the DR to raise the temperature of the OT $100 \mathrm{mK}$ stage to the fiducial operating temperature of $\sim 100 \mathrm{mK}$. As can be seen in Figure 15, the cold plate temperature varied by $\lesssim 0.25 \mathrm{mK}$ over the (representative) 48 $\mathrm{hr}$ period. At this level, bath temperature fluctuations will have negligible impact on detector stability. The thermal gradient across the OT $100 \mathrm{mK}$ stage was measured to be $\lesssim 1 \mathrm{mK}$ at base temperature. A gradient this small means that adjacent detector arrays within a single OT will have negligible differences in bath temperature, preventing degradation of the detector data quality. Thus, these validation test show that we have met the design requirements.

\subsection{Vibration Tests}

An area of concern in the thermal-mechanical design of the receiver is microphonic heating of the coldest stages, and particularly of the detector arrays, by vibrational sources in the cryostat and the receiver cabin (Bhatia et al. 1996). In addition, vibration pickup can add to detector noise. Predicting the magnitude of this loading prior to the construction of the receiver is very difficult, and moreover the heating is most noticeable at the $1 \mathrm{~K}$ and $100 \mathrm{mK}$ stages. FEA can provide some leverage in the design phase by allowing us to predict which components of the $1 \mathrm{~K}$ and $100 \mathrm{mK}$ stages will have low-frequency fundamental vibration modes that are more likely to couple to the vibrational environment of the receiver. These simulations were performed, and are detailed in Orlowski-Scherer et al. (2018). Ultimately, however, vibrational FEA of the entire receiver is not feasible, and the FEA contains no information about the amplitude of the vibrations. Therefore, we need to test the transfer function of the receiver in the lab.

To test the vibration sensitivity of the $100 \mathrm{mK}$ stages, we attached a Buttkicker mini Concert transducer ${ }^{53}$ to the front flange of the cryostat, driven using its associated amplifier with $\sim 200 \mathrm{~W}$ of power. The $100 \mathrm{mK}$ stage was below $100 \mathrm{mK}$ when we performed the test. We tested attaching the Buttkicker to the middle and back flanges and found it made no significant difference between the two configurations. We used a tone generator to supply the signal to the amplifier. We then monitored the temperature at a number of points on the $100 \mathrm{mK}$ stages while slowly sweeping the input tone. By carefully recording when we started the sweep, the speed of the sweep, and the times at which we observed spikes in the temperatures, we could determine the frequency at the time of the spike, which we interpreted as a resonance frequency somewhere in the system. We ran the sweeps both increasing and decreasing in frequency as a consistency check and to account for any time delays in the temperature response. Once we identified a resonance, we would sweep very slowly over the resonance to accurately recover its frequency. In general, all these tests agreed to within about $1 \mathrm{~Hz}$. Further, by observing the magnitude of the temperature rise at a number of locations, we could roughly localize the source of the heating and infer which part of the cryostat was strongly coupled to that vibration. By this method, we have determined that the lowest harmonics of the $100 \mathrm{mK}$ stage are at $21,24,27$, and $31 \mathrm{~Hz}$, all of which are associated with the structure at or near the OT 100 $\mathrm{mK}$ cold finger. The cold finger shown in Figure 9 is a long and thin rigid body, so it is particularly susceptible to microphonic heating. The resonance at $24 \mathrm{~Hz}$ is by far the largest in amplitude, at approximately seven times larger temperature gain than the others. While current calculations indicate this heating mode will not affect the performance of the receiver, avenues for stiffening the part are being explored.

\section{Future Development}

Although the tests described in this paper demonstrate that the LATR meets all requirements, there are some areas where the system performance could be further optimized. Some of them were overdesigned, meaning we could have achieved the same performance without sacrificing performance on other aspects; on the other hand, some of the parts were underdesigned, meaning the performance could be boosted significantly if we had given more margins in the design. We hope that the lessons learned here will be beneficial for future experiments developing large-volume ultracold cryostats, for example CMB-S4 (Abazajian et al. 2016, 2019; Abitbol et al. 2017).

The $40 \mathrm{~K}$ stage, running from the very back to the very front of the cryostat, is the most spatially extended stage in the LATR. The shells in this stage are responsible for conducting the cooling power from the pulse tube cold heads, which are mounted at the midpoint of the stage. The initial design called for the $40 \mathrm{~K}$ shell to be made of $1 / 8$ inch aluminum 1100 series, which proved to be not thick enough. It caused a gradient concentrated around the location where the pulse tubes are attached to the $40 \mathrm{~K}$ shell. The thermal conductivity was improved by installing additional $1 / 4-1 / 2$ inch thick

\footnotetext{
${ }^{53}$ Buttkicker https://thebuttkicker.com/.
} 
high-purity (99.999\%) aluminum cladding on the $40 \mathrm{~K}$ shells. The modification significantly reduced the thermal gradient on the $40 \mathrm{~K}$ stage. In hindsight, it would have been wise to leave extra margins for thermal conductivity, particularly on a stage that spatially spans the entire cryostat.

More generally, a lesson we learned from developing and testing the LATR is that distributing the cooling power is more important than adding cooling power. For future large cryostat development efforts, evenly distributing the cooling power by boosting the thermal conductivity between different parts should be a high priority in the cryogenic design. With optimized thermal connections between temperature stages, not only will thermal gradients be small, but the cooldown time will be significantly reduced, removing the need for other complicated precooling systems.

A significant amount of effort went into minimizing the number of bolted joints on $1 \mathrm{~K}$ and $100 \mathrm{mK}$ stages, in order to reduce the temperature gradient on these two stages. We accomplished this by welding vertical rods onto the $1 \mathrm{~K}$ and $100 \mathrm{mK}$ thermal BUSs to reach as close to the OT $1 \mathrm{~K}$ and $100 \mathrm{mK}$ cold fingers as possible. While the $1 \mathrm{~K}$ BUS performed well enough to meet the specification, the $100 \mathrm{mK}$ BUS suffered from unreliable welding quality, which resulted in a large temperature gradient between the OT focal plane base plate and the cryostat's $100 \mathrm{mK}$ BUS. To remedy this, we chose to drill new holes and installed bolted rods on the $100 \mathrm{mK}$ BUS, which performed much better than the welded rods. Due to the size of the $100 \mathrm{mK}$ BUS, a large amount of heat is required for the welding process, which can lead to warping and difficulty controlling the quality of the welded joints. For future applications, we would recommend employing bolted joints for ease of manufacture and installation.

Given the results of the pull test of the glue joint, for future development we would recommend changing the design of the glue joints. In our current design, our G-10 tabs are inserted into a U-shaped cavity with prefilled glue and then left to cure. This design has the advantage of simplicity in manufacture and assembly, but has the significant disadvantage of relying on the bond strength of the glue. Indeed, in all pull testing we performed, the glue failure was adhesive, specifically from the aluminum walls of the cavity. Instead, we recommend a "pocket-cavity" design, wherein a pocket is machined in the foot, with a tight clearance hole for the G10 at the pocket entrance. Injection holes around the sides provide access to the cavity for gluing. This design is more complicated to manufacture but has the advantage that the glue can no longer fail in adhesion from the aluminum due to the "roof" of the cavity. An example of this design is shown in Figure 15. While we did not switch the design of the G-10 tabs, as they were already installed when we developed this design, we did successfully use a similar design for the $1 \mathrm{~K}-100 \mathrm{mK}$ standoffs.

\section{Conclusion}

The Simons Observatory (SO) Large Aperture Telescope Receiver (LATR) will be installed in the Large Aperture Telescope (LAT) that utilizes a cross-Dragone design with two $6 \mathrm{~m}$ aperture mirrors. The LATR contains five cryogenic stages, including $80 \mathrm{~K}, 40 \mathrm{~K}, 4 \mathrm{~K}, 1 \mathrm{~K}$, and the ultimate $100 \mathrm{mK}$ stage where detectors operate. Various cryogenic optics, including optical filters and cryogenic lenses, are installed on the cryogenic stages to achieve designed thermal and optical performances. At its heart, the LATR is capable of cooling $>62,000$ transition edge sensors (TES; Irwin \& Hilton 2005) to below $100 \mathrm{mK}$, with a sub-mK long-term temperature stability. At this temperature, detector noise is subdominant to incoming photon shot noise.

The detectors and the $\leqslant 4 \mathrm{~K}$ optics are packaged in modular units called optics tubes (OTs). The modularized design facilitates efficient installation and removal without sacrificing precise control of the relative positions of the optics. Our initial plan is to deploy with seven OTs in the nominal LATR configuration, with a potential upgrade to 13 OTs in the future. Carrying detector data out of the cryostat requires hundreds of coaxial cables and thousands of cryogenic wires. To organize all the cables and make it easily serviceable, the SO developed the universal readout harness to modularize the $300 \mathrm{~K}-40 \mathrm{~K}$ wiring.

The LATR has been constructed and extensively tested. We started with the simplest configuration and gradually added more factors. Currently, the LATR has been tested with three optical windows, one OT, and two feedthroughs installed (one for detector signals and one for housekeeping). All of the five cryogenic stages cool to their base temperatures within five days (the cooldown time will become longer as the number of OT increases), with the anticipated thermal loading and thermal gradient on each temperature stage. Extrapolating from these partially equipped results, the LATR will perform as designed when all the 13 OTs and 5 feedthroughs are installed.

The development of the LATR offers critical insight and experience on sub-Kelvin cryostats at this unprecedented size, which will be valuable for future $\mathrm{CMB}$ experiments, including CMB-S4 (Abazajian et al. 2016; Abitbol et al. 2017).

We thank the Vertex ${ }^{54}$ group, who have been working closely with us on the telescope and receiver interface design. Dynavac, ${ }^{55}$ Meyer Tool, ${ }^{56}$ PVEng, ${ }^{57}$ and Fermilab cryogenic engineering groups have provided a number of helpful suggestions regarding the cryostat design and manufacture process. We also thank Jeffrey Hancock and Harold Borders from the University of Pennsylvania Physics machine shop.

This work was supported in part by a grant from the Simons Foundation (award \#457687, B.K.). We acknowledge support from the JSPS KAKENHI grant No. JP17H06134. Zhilei Xu is supported by the Gordon and Betty Moore Foundation.

\section{ORCID iDs}

Ningfeng Zhu (1D https://orcid.org/0000-0002-3037-2003

Tanay Bhandarkar (iD https://orcid.org/0000-0002-2971-1776

John L. Orlowski-Scherer (i) https://orcid.org/0000-00031842-8104

Zhilei Xu (iD https://orcid.org/0000-0001-5112-2567

Peter Ade (iD https://orcid.org/0000-0002-5127-0401

Simone Aiola (i) https://orcid.org/0000-0002-1035-1854

Steve K. Choi (iD https://orcid.org/0000-0002-9113-7058

Jake Connors (iD https://orcid.org/0000-0002-6094-2943

Nicholas F. Cothard (iD https://orcid.org/0000-0002-

6151-6292

Mark Devlin (1D https://orcid.org/0000-0002-3169-9761

Simon Dicker (ib https://orcid.org/0000-0002-1940-4289

\footnotetext{
54 Vertex Antennentechnik GmbH, https://www.vertexant.com/.

55 https://www.dynavac.com/

56 https://www.meyertool.com/

57 Pressure Vessel Engineering Limited, https://www.pveng.com/.
} 
Bradley Dober (다 https://orcid.org/0000-0002-6817-0829

Shannon M. Duff (1) https://orcid.org/0000-0002-9693-4478

Rolando Dünner (1) https://orcid.org/0000-0003-3892-1860

Giulio Fabbian (ํ) https://orcid.org/0000-0002-3255-4695

Nicholas Galitzki (i) https://orcid.org/0000-0001-7225-6679

Patricio A. Gallardo (1) https://orcid.org/0000-0001-

9731-3617

Saianeesh K. Haridas 주 https://orcid.org/0000-0001-

6519-502X

Kathleen Harrington (10) https://orcid.org/0000-0003-

1248-9563

Zachary B. Huber @ https://orcid.org/0000-0003-4573-4094

Jeffrey Iuliano (1) https://orcid.org/0000-0001-7466-0317

Bradley R. Johnson (10) https://orcid.org/0000-0002-6898-8938

Kenji Kiuchi iㅏ https://orcid.org/0000-0003-4988-1438

Brian J. Koopman (1) https://orcid.org/0000-0003-0744-2808

Michele Limon (1) https://orcid.org/0000-0002-5900-2698

Tammy J Lucas (1) https://orcid.org/0000-0001-7694-1999

Jenna Moore (ib https://orcid.org/0000-0002-7340-9291

Federico Nati (i) https://orcid.org/0000-0002-8307-5088

Michael D. Niemack @ https://orcid.org/0000-0001-

7125-3580

Jacob Spisak iㅏ https://orcid.org/0000-0003-1789-8550

Tran Tsan $\odot$ https://orcid.org/0000-0002-1667-2544

Carole Tucker (1) https://orcid.org/0000-0002-1851-3918

Michael R. Vissers (1) https://orcid.org/0000-0003-2467-7801

Samantha Walker (1) https://orcid.org/0000-0002-5855-4036

Edward J. Wollack (i) https://orcid.org/0000-0002-7567-4451

Mario Zannoni (i) https://orcid.org/0000-0002-4495-571X

\section{References}

Abazajian, K., Addison, G., Adshead, P., et al. 2019, arXiv:1907.04473 Abazajian, K. N., Adshead, P., Ahmed, Z., et al. 2016, arXiv:1610.02743 Abitbol, M. H., Ahmed, Z., Barron, D., et al. 2017, arXiv:1706.02464 Ade, P., Ahmed, Z., Amiri, M., et al. 2021, PhRvD, 103, 022004 Ade, P., Aikin, R., Barkats, D., et al. 2014a, PhRvL, 112, 241101 Ade, P., Akiba, Y., Anthony, A., et al. 2014b, PhRvL, 113, 021301 Ade, P. A. R., Pisano, G., Tucker, C., \& Weaver, S. 2006, Proc. SPIE, 6275, $62750 \mathrm{U}$

Alduino, C., Alessandria, F., Balata, M., et al. 2019, Cryo, 102, 9

Ali, A. M., Adachi, S., Arnold, K., et al. 2020, JLTP, 200, 461

Baxter, E. J., Jain, B., Blake, C., et al. 2018, arXiv:1812.08701

Bennett, C. L., Larson, D., Weiland, J. L., et al. 2013, ApJS, 208, 20

Bhatia, R. S., Ade, P. A. R., Griffin, M. J., Hepburn, I. D., \& Murray, A. G. 1996, ESASP, 388, 277

Blake, C., Baxter, E., \& Jain, B. 2019, AAS Meeting Abstracts, 233, 302.06

Carlstrom, J. E., Ade, P. A. R., Aird, K. A., et al. 2011, PASP, 123, 568

Choi, S. K., Hasselfield, M., Ho, S.-P. P., et al. 2020, JCAP, 2020, 045

Condon, J. J., \& Matthews, A. M. 2018, PASP, 130, 073001

Coppi, G., Xu, Z., Ali, A., et al. 2018, Proc. SPIE, 10708, 1070827

Coughlin, K. P., McMahon, J. J., Crowley, K. T., et al. 2018, JLTP, 193, 876

Datta, R., Munson, C. D., Niemack, M. D., et al. 2013, ApOpt, 52, 8747

DESI Collaboration, Aghamousa, A., Aguilar, J., et al. 2016, arXiv:1611.00036

Dicker, S. R., Gallardo, P. A., Gudmundsson, J. E., et al. 2018, Proc. SPIE, 10700, 107003E

Dober, B., Ahmed, Z., Arnold, K., et al. 2021, ApPhL, 118, 062601

Duband, L., Prouve, T., Bock, J., Moncelsi, L., \& Schillaci, A. 2018, Proc. 20th Int. Cryocooler Conf., ed. S. D. Miller et al. (Boulder, CO: International Cryocooler Conference, Inc.),347, https://cryocooler.org/ resources/Documents/C20/347.pdf\#page $=1$

Duff, S. M., Austermann, J., Beall, J. A., et al. 2016, JLTP, 184, 634

Galitzki, N., Ali, A., Arnold, K. S., et al. 2018, Proc. SPIE, 10708, 1070804

Gallardo, P. A., Gudmundsson, J., Koopman, B. J., et al. 2018, Proc. SPIE, $10708,107083 \mathrm{Y}$
Golec, J. E., McMahon, J. J., Ali, A., et al. 2020, Proc. SPIE, 11451, 1182 Gualtieri, R., Filippini, J. P., Ade, P. A. R., et al. 2018, JLTP, 193, 1112

Gudmundsson, J., Ade, P., Amiri, M., et al. 2015, Cryo, 72, 65

Gudmundsson, J. E., Gallardo, P. A., Puddu, R., et al. 2021, ApOpt, 60, 823

Guns, S., Foster, A., Daley, C., et al. 2021, ApJ, 916, 98

Harrington, K., Marriage, T., Ali, A., et al. 2016, Proc. SPIE, 9914, 99141K

Harrington, K., Sierra, C., Chesmore, G., et al. 2020, Proc. SPIE, 11453, 1145318

Henderson, S. W., Allison, R., Austermann, J., et al. 2016, JLTP, 184, 772

Hill, C. A., Bruno, S. M. M., Simon, S. M., et al. 2018, Proc. SPIE, 10708, 1070842

Hilton, M., Hasselfield, M., Sifón, C., et al. 2018, ApJS, 235, 20

Hinshaw, G., Larson, D., Komatsu, E., et al. 2013, ApJS, 208, 19

Hu, W., \& White, M. 1997, NewA, 2, 323

Irwin, K. D., \& Hilton, G. C. 2005, in Cryogenic Particle Detection, ed. C. Enss (Berlin: Springer), 63

Ivezić, Ž., Kahn, S. M., Tyson, J. A., et al. 2019, ApJ, 873, 111

Keck Array and BICEP2 Collaborations, Ade, P. A. R., Ahmed, Z., et al. 2018, PhRvL, 121, 221301

Keller, C. W., Cunnington, G. R., \& Glassford, A. P. 1974, Thermal Performance of Multilayer Insulations Final Report, NASA CR-72747/ LMSC-A974469, Lockheed Missiles \& Space Company, https://ntrs.nasa. gov/api/citations/19710014823/downloads/19710014823.pdf

Koopman, B. J., Lashner, J., Saunders, L. J., et al. 2020, Proc. SPIE, 11452, 1145208

Kusaka, A., Appel, J., Essinger-Hileman, T., et al. 2018, JCAP, 2018, 005

Li, Y., Arnold, K., Atkins, Z., et al. 2020, JLTP, 199, 985

Lizon, J. L., \& Accardo, M. 2010, Proc. SPIE, 7739, 77393E

Marquardt, E., Le, J., \& Radebaugh, R. 2002, Cryocoolers 11 (Berlin: Springer), 681

Mates, J. A. B., Irwin, K. D., Vale, L. R., et al. 2012, JLTP, 167, 707

Mather, J. C. 1984, ApOpt, 23, 584

McCarrick, H., Healy, E., Ahmed, Z., et al. 2021, ApJ, in press, arXiv:2106. 14797

Nadolski, A., Kofman, A. M., Vieira, J. D., et al. 2018, Proc. SPIE, 10708 1070843

Naess, S., Battaglia, N., Bond, J. R., et al. 2021, ApJ, 915, 14

NIST 2009, Index of Material Properties, https://trc.nist.gov/cryogenics/ materials/materialproperties.htm

Orlowski-Scherer, J., Baxter, E., Devlin, M., Jain, B., \& Blake, C. 2019 , BAAS, 51, 349

Orlowski-Scherer, J. L., Zhu, N., Xu, Z., et al. 2018, Proc. SPIE, 10708 107083X

Pan, Z., Ade, P. A. R., Ahmed, Z., et al. 2018, JLTP, 193, 305

Parshley, S. C., Niemack, M., Hills, R., et al. 2018, Proc. SPIE, 10700 , 1070041

Planck Collaboration, Aghanim, N., Akrami, Y., et al. 2020, A\&A, 641, A5

Polarbear Collaboration, Adachi, S., Aguilar Faúndez, M. A. O., et al. 2020, ApJ, 897, 55

Posada, C. M., Ade, P. A. R., Ahmed, Z., et al. 2015, SuScT, 28, 094002

Sathyanarayana Rao, M., Silva-Feaver, M., Ali, A., et al. 2020, JLTP, 199,807

Sayre, J. T., Reichardt, C. L., Henning, J. W., et al. 2020, PhRvD, 101, 122003

Smoot, G. F. 1999, in AIP Conf. Proc. 476, Conf. on 3K Cosmology (Melville, NY: AIP), 1

Story, K. T., Hanson, D., Ade, P. A. R., et al. 2015, ApJ, 810, 50

Sunyaev, R. A., \& Zeldovich, Y. B. 1970, CoASP, 2, 66

Sunyaev, R. A., \& Zeldovich, Y. B. 1972, CoASP, 4, 173

Suzuki, A., Ade, P., Akiba, Y., et al. 2016, JLTP, 184, 805

The Simons Observatory Collaboration 2019, JCAP, 2019, 056

Thornton, R. J., Ade, P. A. R., Aiola, S., et al. 2016, ApJS, 227, 21

Tucker, C. E., \& Ade, P. A. R. 2006, Proc. SPIE, 6275, 62750T

Vavagiakis, E. M., Ahmed, Z., Ali, A., et al. 2020, arXiv:2012.04532

Woodcraft, A. L., \& Gray, A. 2009, in AIP Conf. Ser. 1185, The Thirteenth Int. Workshop on Low Temperature Detectors, ed. B. Young, B. Cabrera, \& A. Miller (Melville, NY: AIP), 681

Wu, W. L. K., Mocanu, L. M., Ade, P. A. R., et al. 2019, ApJ, 884, 70

Xu, Z., Bhandarkar, T., Coppi, G., et al. 2020, Proc. SPIE, 11453, 207

Xu, Z., Brewer, M. K., Rojas, P. F., et al. 2020, ApJ, 891, 134

Xu, Z., Chesmore, G. E., Adachi, S., et al. 2021, ApOpt, 60, 864

Zhu, N., Orlowski-Scherer, J. L., Xu, Z., et al. 2018, Proc. SPIE, 10708, 259 


\section{Erratum: "The Simons Observatory Large Aperture Telescope Receiver" (2021, ApJS, 256, 23)}

Ningfeng Zhu ${ }^{1}$ (D), Tanay Bhandarkar ${ }^{1}$ (D) , Gabriele Coppi ${ }^{1,2}$, Anna M. Kofman ${ }^{1}$ (D), John L. Orlowski-Scherer ${ }^{1}$ (D), Zhilei Xu ${ }^{1,3}$ (D), Shunsuke Adachi ${ }^{4}$, Peter Ade ${ }^{5}$, Simone Aiola ${ }^{6}$ (D), Jason Austermann ${ }^{7}$ (D), Andrew O. Bazarko ${ }^{8}$, James A. Beall ${ }^{7}$, Sanah Bhimani ${ }^{9}$, J. Richard Bond ${ }^{10}$ (D), Grace E. Chesmore ${ }^{11}$, Steve K. Choi ${ }^{12,13}$, Jake Connors ${ }^{7}$ (D), Nicholas F. Cothard ${ }^{14}$ (D), Mark Devlin ${ }^{1}$, Simon Dicker ${ }^{1}$ (D) , Bradley Dober ${ }^{15}$ (D) , Cody J. Duell ${ }^{12}$, Shannon M. Duff ${ }^{7}$ (D), Rolando Dünner ${ }^{16}$ (D), Giulio Fabbian ${ }^{5}$ (iD), Nicholas Galitzki $^{17}$ (D), Patricio A. Gallardo ${ }^{12}$ (D) , Joseph E. Golec ${ }^{11}$, Saianeesh K. Haridas ${ }^{1}$ (D), Kathleen Harrington ${ }^{18}$ (iD), Erin Healy ${ }^{8}$ (D), Shuay-Pwu Patty Ho ${ }^{19}$, Zachary B. Huber ${ }^{12}$ (D) , Johannes Hubmayr ${ }^{7}$, Jeffrey Iuliano ${ }^{1}$ (D), Bradley R. Johnson ${ }^{20}$ (D), Brian Keating $^{17}$ (D), Kenji Kiuchi ${ }^{21}$, Brian J. Koopman ${ }^{9}$ (D), Jack Lashner ${ }^{22}$, Adrian T. Lee ${ }^{23}$, Yaqiong Li ${ }^{12,24}$, Michele Limon ${ }^{1}$ (iD), Michael Link ${ }^{7}$, Tammy J Lucas ${ }^{7}$ (D), Heather McCarrick ${ }^{8}$ (D), Jenna Moore ${ }^{25}$ (D), Federico Nati ${ }^{2}$ (D), Laura B. Newburgh ${ }^{26}$, Michael D. Niemack ${ }^{12,13,24}$ (D), Elena Pierpaoli ${ }^{22}$, Michael J. Randall ${ }^{17}$, Karen Perez Sarmiento ${ }^{1}$, Lauren J. Saunders ${ }^{9}$, Joseph Seibert $^{17}$, Carlos Sierra ${ }^{11}$, Rita Sonka ${ }^{8}$, Jacob Spisak ${ }^{17}$ (D), Shreya Sutariya ${ }^{11}$, Osamu Tajima ${ }^{4}$, Grant P. Teply ${ }^{17}$, Robert J. Thornton ${ }^{1,27}$ (D), Tran Tsan ${ }^{17}$ (D), Carole Tucker $^{5}$ (D), Joel Ullom ${ }^{7}$, Eve M. Vavagiakis ${ }^{12}$, Michael R. Vissers ${ }^{7}$ (D), Samantha Walker ${ }^{7,28}$ (D) , Benjamin Westbrook ${ }^{23}$, Edward J. Wollack ${ }^{29}$ (D), and Mario Zannoni ${ }^{2}$ (D)

${ }^{1}$ Department of Physics and Astronomy, University of Pennsylvania, 209 South 33rd Street, Philadelphia, PA 19104, USA; nin@sas.upenn.edu

${ }^{2}$ Department of Physics, University of Milano-Bicocca, Milano (MI), Italy

${ }^{3}$ MIT Kavli Institute, Massachusetts Institute of Technology, 77 Massachusetts Avenue, Cambridge, MA 02139, USA

${ }^{4}$ Department of Physics, Kyoto University, Kitashirakawa Oiwake-cho, Sakyo-ku, Kyoto 606-8502, Japan

${ }^{5}$ School of Physics and Astronomy, Cardiff University, The Parade, Cardiff, CF24 3AA, UK

${ }^{6}$ Center for Computational Astrophysics, Flatiron Institute, 162 5th Avenue, New York, NY 10010, USA ${ }^{7}$ NIST Quantum Sensors Group, 325 Broadway Ave, Boulder, CO 80305, USA

${ }^{8}$ Department of Physics, Princeton University, Princeton, NJ 08540, USA

${ }^{9}$ Department of Physics, Yale University, New Haven, CT 06520, USA

${ }^{10}$ CITA, University of Toronto, Toronto, ON M5S 3H8 Canada

${ }^{11}$ Department of Physics, University of Chicago, Chicago, IL, 60637, USA

${ }_{13}^{12}$ Department of Physics, Cornell University, Ithaca, NY 14853, USA

${ }^{13}$ Department of Astronomy, Cornell University, Ithaca, NY 14853, USA

${ }^{14}$ Department of Applied and Engineering Physics, Cornell University, Ithaca, NY 14853, USA

15 Department of Physics, University of Colorado Boulder, Department of Physics 390, Boulder, CO 80309, USA

${ }^{16}$ Instituto de Astrofísica and Centro de Astro-Ingeniería, Facultad de Física, Pontificia Universidad Católica de Chile, Av. Vicuña Mackenna 4860, 7820436, Macul, Santiago, Chile

${ }^{17}$ Department of Physics, University of California San Diego, La Jolla, CA 92093, USA

${ }^{18}$ Department of Astronomy and Astrophysics, University of Chicago, Chicago, IL, 60637, USA

${ }^{19}$ Department of Physics, Stanford University, Stanford, CA 94305, USA

${ }^{20}$ Department of Astronomy, University of Virginia, Charlottesville, VA 22904, USA

${ }^{21}$ Department of Physics, The University of Tokyo, 7-3-1 Hongo, Bunkyo, Tokyo 113-0033, Japan

22 Department of Physics and Astronomy, University of Southern California, Los Angeles, CA 90089, USA

${ }^{23}$ Physics Department, University of California, Berkeley, CA 94720, USA

${ }^{24}$ Kavli Institute at Cornell for Nanoscale Science, Cornell University, Ithaca, NY 14853, USA

${ }^{25}$ School of Earth and Space Exploration, Arizona State University, Tempe, AZ 85287, USA

${ }^{26}$ Department of Physics, Yale University, New Haven, CT 06511, USA

${ }^{27}$ Department of Physics and Engineering, West Chester University of Pennsylvania, West Chester, PA 19383, USA

${ }^{28}$ Department of Astrophysical and Planetary Sciences, University of Colorado Boulder, Boulder, CO 80309, USA

${ }^{29}$ NASA Goddard Space Flight Center, Greenbelt, MD 20771, USA

Received 2021 October 8; published 2021 December 10

\section{Erratum}

After the publication of the article, it was brought to our attention that the description of Equation (1) may cause potential confusion. Thus, we have decided to provide a newer reference and added a unit for $q_{\text {tot }}$ in the description. The updated paragraph should read as the following:

The thermal conduction due to radiation on surfaces covered in MLI was computed using the Lockhead equation (Ross 2015):

$$
\begin{aligned}
q_{\mathrm{tot}}= & \frac{C_{c} N^{2.56} T_{m}}{n}\left(T_{h}-T_{c}\right) \\
& +\frac{C_{r} \epsilon_{0}}{n}\left(T_{h}^{4.67}-T_{c}^{4.67}\right),
\end{aligned}
$$

which accounts for both the conductive loading through the layers of the blanket (first term), and the radiative loading between layers of the MLI blanket (second term). In Equation (1), $q_{\mathrm{tot}}$ is the total thermal load on the MLI in units 
of $\mathrm{mW} \mathrm{m}^{-2}, C_{c}=8.95 \times 10^{-5}$ is a numerical constant defining the MLI conductive heat transfer, $N$ is the MLI layer density in layers per centimeter, $T_{m}$ is the mean MLI temperature, taken to be $T_{m}=\frac{T_{h}-T_{c}}{2}, n$ is the number of MLI layers, $T_{h}$ is the hot-side temperature in kelvin, $T_{c}$ is the cold-side temperature in kelvin, $C_{r}=5.39 \times 10^{-7}$ is a numerical constant defining the MLI radiative heat transfer, and $\epsilon_{0}=0.031$ is the MLI emissivity (Ross 2015).

\section{ORCID iDs}

Ningfeng Zhu (10 https://orcid.org/0000-0002-3037-2003

Tanay Bhandarkar (1) https://orcid.org/0000-0002-2971-1776

Anna M. Kofman (ํ) https://orcid.org/0000-0001-5374-1767

John L. Orlowski-Scherer (1) https://orcid.org/0000-0003-

1842-8104

Zhilei Xu (1) https://orcid.org/0000-0001-5112-2567

Simone Aiola (1) https://orcid.org/0000-0002-1035-1854

Jason Austermann (1) https://orcid.org/0000-0002-6338-0069

J. Richard Bond (il https://orcid.org/0000-0003-2358-9949

Jake Connors (1) https://orcid.org/0000-0002-6094-2943

Nicholas F. Cothard (1) https://orcid.org/0000-0002-

6151-6292

Simon Dicker (1) https://orcid.org/0000-0002-1940-4289

Bradley Dober (1) https://orcid.org/0000-0002-6817-0829

Shannon M. Duff (1) https://orcid.org/0000-0002-9693-4478

Rolando Dünner (1) https://orcid.org/0000-0003-3892-1860

Giulio Fabbian (1) https://orcid.org/0000-0002-3255-4695

Nicholas Galitzki (1) https://orcid.org/0000-0001-7225-6679

Patricio A. Gallardo (ib https://orcid.org/0000-0001-

9731-3617

Saianeesh K. Haridas ํํ https://orcid.org/0000-00016519-502X

Kathleen Harrington (17) https://orcid.org/0000-00031248-9563

Erin Healy (1) https://orcid.org/0000-0002-3757-4898

Zachary B. Huber (ii) https://orcid.org/0000-0003-4573-4094

Jeffrey Iuliano (i) https://orcid.org/0000-0001-7466-0317

Bradley R. Johnson (i) https://orcid.org/0000-0002-6898-8938

Brian Keating (1) https://orcid.org/0000-0003-3118-5514

Brian J. Koopman (10 https://orcid.org/0000-0003-0744-2808

Michele Limon (ㄴ) https://orcid.org/0000-0002-5900-2698

Tammy J Lucas (1) https://orcid.org/0000-0001-7694-1999

Heather McCarrick (1) https://orcid.org/0000-0002-5490-4211

Jenna Moore (ii https://orcid.org/0000-0002-7340-9291

Federico Nati (1) https://orcid.org/0000-0002-8307-5088

Michael D. Niemack @ https://orcid.org/0000-0001-

7125-3580

Jacob Spisak iㅏ https://orcid.org/0000-0003-1789-8550

Robert J. Thornton (1) https://orcid.org/0000-0002-3180-674X

Tran Tsan (ib https://orcid.org/0000-0002-1667-2544

Carole Tucker (1) https://orcid.org/0000-0002-1851-3918

Michael R. Vissers (i) https://orcid.org/0000-0003-2467-7801

Samantha Walker (i) https://orcid.org/0000-0002-5855-4036

Edward J. Wollack (1) https://orcid.org/0000-0002-7567-4451

Mario Zannoni (i) https://orcid.org/0000-0002-4495-571X

\section{References}

